\title{
Isocyanogen formation in the cold interstellar medium
}

\author{
C. Vastel ${ }^{1}$, J. C. Loison ${ }^{2}$, V. Wakelam ${ }^{3}$, and B. Lefloch ${ }^{4}$ \\ ${ }^{1}$ IRAP, Université de Toulouse, CNRS, UPS, CNES, 31400 Toulouse, France \\ e-mail: cvastel@irap.omp.eu \\ ${ }^{2}$ Institut des Sciences Moléculaires (ISM), CNRS, Université de Bordeaux, 351 cours de la Libération, 33400 Talence, France \\ ${ }^{3}$ Laboratoire d'astrophysique de Bordeaux, Université de Bordeaux, CNRS, B18N, allée Geoffroy Saint-Hilaire, 33615 Pessac, \\ France \\ ${ }^{4}$ CNRS, IPAG, Université Grenoble Alpes, 38000 Grenoble, France
}

Received 3 January 2019 / Accepted 23 March 2019

\begin{abstract}
Context. Cyanogen (NCCN) is the simplest member of the dicyanopolyynes group, and has been proposed as a major source of the $\mathrm{CN}$ radical observed in cometary atmospheres. Although not detected through its rotational spectrum in the cold interstellar medium, this very stable species is supposed to be very abundant.

Aims. The chemistry of cyanogen in the cold interstellar medium can be investigated through its metastable isomer, CNCN (isocyanogen). Its formation may provide a clue on the widely abundant $\mathrm{CN}$ radical observed in cometary atmospheres.

Methods. We performed an unbiased spectral survey of the L1544 proto-typical prestellar core, using the IRAM- $30 \mathrm{~m}$ and have analysed, for this paper, the nitrogen chemistry that leads to the formation of isocyanogen. We report on the first detection of $\mathrm{CNCN}$, $\mathrm{NCCNH}^{+}, \mathrm{C}_{3} \mathrm{~N}, \mathrm{CH}_{3} \mathrm{CN}, \mathrm{C}_{2} \mathrm{H}_{3} \mathrm{CN}$, and $\mathrm{H}_{2} \mathrm{CN}$ in L1544. We built a detailed chemical network for NCCN/CNCN/HC $2 \mathrm{~N}_{2}^{+}$involving all the nitrogen bearing species detected $\left(\mathrm{CN}, \mathrm{HCN}, \mathrm{HNC}, \mathrm{C}_{3} \mathrm{~N}, \mathrm{CNCN}, \mathrm{CH}_{3} \mathrm{CN}, \mathrm{CH}_{2} \mathrm{CN}, \mathrm{HCCNC}, \mathrm{HC}_{3} \mathrm{~N}, \mathrm{HNC}_{3}, \mathrm{H}_{2} \mathrm{CN}, \mathrm{C}_{2} \mathrm{H}_{3} \mathrm{CN}\right.$, $\mathrm{HCNH}^{+}, \mathrm{HC}_{3} \mathrm{NH}+$ ) and the upper limits on $\mathrm{C}_{4} \mathrm{~N}, \mathrm{C}_{2} \mathrm{~N}$. The main cyanogen production pathways considered in the network are the $\mathrm{CN}+\mathrm{HNC}$ and $\mathrm{N}+\mathrm{C}_{3} \mathrm{~N}$ reactions.

Results. The comparison between the observations of the nitrogen bearing species and the predictions from the chemical modelling shows a very good agreement, taking into account the new chemical network. The expected cyanogen abundance is greater than the isocyanogen abundance by a factor of 100 . Although cyanogen cannot be detected through its rotational spectrum, the chemical modelling predicts that it should be abundant in the gas phase and hence might be traced through the detection of isocyanogen. It is however expected to have a very low abundance on the grain surfaces compared to HCN.
\end{abstract}

Key words. astrochemistry - molecular processes - line: identification - molecular data - radiative transfer

\section{Introduction}

Molecular complexity in the cold interstellar medium has been revealed to be extremely rich, due to the sensitivity and spectral resolution of modern instruments. Organic molecules have been detected, such as alcohols, aldehydes, acids, ethers, amines, and nitriles. The strong bond of the cyano group $(-\mathrm{C} \equiv \mathrm{N})$ is present in many species such as cyanopolyynes $\left(\mathrm{HC}_{n} \mathrm{~N}\right.$ with $\left.n=3,5,7, \ldots\right)$, and it has also been speculated that dicyanopolyynes might be abundant in interstellar clouds (Kolos \& Grabowski 2000; Petrie et al. 2003). Dicyanopolyynes are very stable species formed by highly unsaturated linear chain of carbon atoms ended by a cyano group at each edge, such as $\mathrm{N} \equiv \mathrm{C}-(\mathrm{C} \equiv \mathrm{C})_{n}-\mathrm{C} \equiv \mathrm{N}$. The simplest form of dicyanopolyynes is the non polar species, cyanogen ( $\mathrm{NCCN}$, also called $\mathrm{C}_{2} \mathrm{~N}_{2}$ ), that cannot be observed through its rotational spectrum. Its metastable isomer, $\mathrm{CNCN}$ has however been detected recently towards L483, which harbours a Class 0 protostar, and tentatively in the cold dark cloud TMC-1 (Agúndez et al. 2018) by stacking of four transitions. The most plausible formation route in cold clouds is the reaction

$$
\begin{aligned}
\mathrm{CN}+\mathrm{HNC} & \rightarrow \mathrm{NCCN}+\mathrm{H}, \\
& \rightarrow \mathrm{CNCN}+\mathrm{H} .
\end{aligned}
$$

The detection of protonated cyanogen $\left(\mathrm{NCCNH}^{+}\right)$in $\mathrm{TMC}-1$ by Agúndez et al. (2015) is also a proof that cyanogen might be an abundant species in the interstellar medium, produced through the protonation of $\mathrm{NCCN}$ with $\mathrm{H}_{3}^{+}$and $\mathrm{HCO}^{+}$:

$\mathrm{XH}^{+}+\mathrm{NCCN} \rightarrow \mathrm{NCCNH}^{+}+\mathrm{X}$,

with $\mathrm{X}=\mathrm{H}_{2}$ and $\mathrm{CO}$. NCCN, which has been detected in Titan atmosphere (Teanby et al. 2006) but not within cometary comae, has been proposed as a major source of the $\mathrm{CN}$ radical observed in cometary atmospheres (Bockelee-Morvan \& Crovisier 1985; Bonev \& Komitov 2000). Indeed, the photolysis of $\mathrm{HCN}, \mathrm{HC}_{3} \mathrm{~N}$, or NCCN could explain the $\mathrm{CN}$ origin. However the $\mathrm{HC}_{3} \mathrm{~N}$ production rate is likely too low to reproduce $\mathrm{CN}$ density profile and the $\mathrm{CN}$ distribution in comets cannot be explained by the HCN photolysis only (Fray et al. 2005). The presence of NCCN in comets is therefore a reliable hypothesis to explain the $\mathrm{CN}$ origin. $\mathrm{CNCN}$ is a good probe to trace the presence of NCCN, since the two are supposed to be linked through reactions (1) and (2).

Some of the precursors of the molecules detected within comets are very likely formed within molecular clouds (see, for example, Geiss et al. 1999; Bockelée-Morvan et al. 2000; Ehrenfreund \& Charnley 2000), and understanding of the formation of $\mathrm{CNCN}$ and NCCN under cold cloud conditions is a crucial step $\mathrm{s}$ the understanding of the molecular complexity in comets. As part of the IRAM- $30 \mathrm{~m}$ Large Program ASAI ${ }^{1}$ (Lefloch et al. 2018), we carried out a highly sensitive, unbiased spectral survey of the molecular emission of the L1544 prestellar

1 Astrochemical Surveys At Iram: http://www . oan.es/asai/ 
core with a high spectral resolution. In the present study we have examined the nitrogen chemistry in this core using the observations of isocyanogen, protonated cyanogen, and cyanoethynyl, used a radiative transfer modelling to determine the observed column densities and compared with the most up-to-date chemical modelling for cyanogen chemistry.

In Sect. 2 we present the observations from the ASAI spectral survey and the line identification for the cyanogen bearing species. Based on the detections and tentative detection, we compute in Sect. 3 the column densities of these species. In Sect. 4 we present a new chemical modelling and confront the results with the observations.

\section{Observations}

The observations for all transitions quoted in Table 1 were performed at the IRAM- $30 \mathrm{~m}$ towards the dust peak emission of the L1544 pre-stellar core $\left(\alpha_{2000}=05^{\mathrm{h}} 04^{\mathrm{m}} 17.21^{\mathrm{s}}\right.$, $\left.\delta_{2000}=25^{\circ} 10^{\prime} 42.8^{\prime \prime}\right)$ in the framework of the ASAI Large Program. Observations at frequencies lower than $80 \mathrm{GHz}$ have been performed in December 2015. All the details of these observations can be found in Vastel et al. (2014) and Quénard et al. (2017a) and line intensities are expressed in units of mainbeam brightness temperature. The frequency range covered by the present spectral survey is $71.74-115.87 \mathrm{GHz}$. We selected transitions with an upper level energy lower than $30 \mathrm{~K}$, and computed the rms over a range of $15 \mathrm{~km} \mathrm{~s}^{-1}$ with a spectral resolution of $50 \mathrm{kHz}$. Some transitions are labelled multiplet or triplet, meaning that the observations do not have the spectral resolution necessary to resolve these transitions or that the hyperfine structure transitions have the same frequencies and slightly different Einstein coefficients. For example, the CNCN 8-7 transition can be decomposed in nine transitions (hyperfine structure due to the quadrupole of the two ${ }^{14} \mathrm{~N}$ nuclei) at the same frequency of $82.784691 \mathrm{GHz}: 8_{7,8}-7_{6,7}, 8_{9,10}-7_{8,9}, 8_{8,8}-7_{7,7}, 8_{9,9}$ $7_{8,8}, 8_{9,8}-7_{8,7}, 8_{8,7}-7_{7,6}, 8_{7,7}-7_{6,6}, 8_{8,9}-7_{7,8}, 8_{7,6}-7_{6,5}$. Another example is the $8(J=17 / 2)-7(J=15 / 2)$ transition of $\mathrm{C}_{3} \mathrm{~N}$ that can be decomposed in three hyperfine transitions: $F=19 / 2$ $17 / 2$ at $79.1510059 \mathrm{GHz}, F=17 / 2-15 / 2$ at $79.1509881 \mathrm{GHz}$ and $F=15 / 2-13 / 2$ at $79.1509863 \mathrm{GHz}$.

The line identification and analysis have been performed using the CASSIS ${ }^{2}$ software (Vastel et al. 2015a). The results from the line fitting take into account the statistical uncertainties accounting for the rms (estimated over a range of $15 \mathrm{~km} \mathrm{~s}^{-1}$ for a spectral resolution of $50 \mathrm{kHz}$ ).

We report the first detections of $\mathrm{CNCN}$ (isocyanogen), $\mathrm{NCCNH}^{+}$(protonated cyanogen), $\mathrm{C}_{3} \mathrm{~N}$ (cyanoethynyl), $\mathrm{H}_{2} \mathrm{CN}$ (N-methaniminyl or methyleneamidogen), A- and $\mathrm{E}-\mathrm{CH}_{3} \mathrm{CN}$ (methyl cyanide also known as acetonitrile or cyanomethane), and $\mathrm{C}_{2} \mathrm{H}_{3} \mathrm{CN}$ (vinyl cyanide, also known as acrylonitrile or propenenitrile, $\mathrm{H}_{2} \mathrm{C}=\mathrm{CH}-\mathrm{C} \equiv \mathrm{N}$ ) in the $\mathrm{L} 1544$ prestellar core shown in Figs. 1-6 respectively. Some transitions of $\mathrm{C}_{2} \mathrm{~N}$ (cyanomethylidyne) and $\mathrm{C}_{4} \mathrm{~N}$ (cyanopropynylidyne) are covered, although not detected, in this spectral survey.

Only the CNCN $J=8-7$ and 10-9 transitions can be considered as detected ( $4.2 \sigma$ for the latter). We have superimposed in red our LTE best fitting solution (see Sect. 3) upon the observed transitions in Fig. 1. The agreement between the predicted intensities and the observations is satisfying except for the $J=9-8$ transition. In order to improve the signal to noise ratio of our CNCN detection and the physical parameter determination, we have stacked the four transitions found in the spectral survey. The

\footnotetext{
2 http://cassis.irap.omp.eu
}

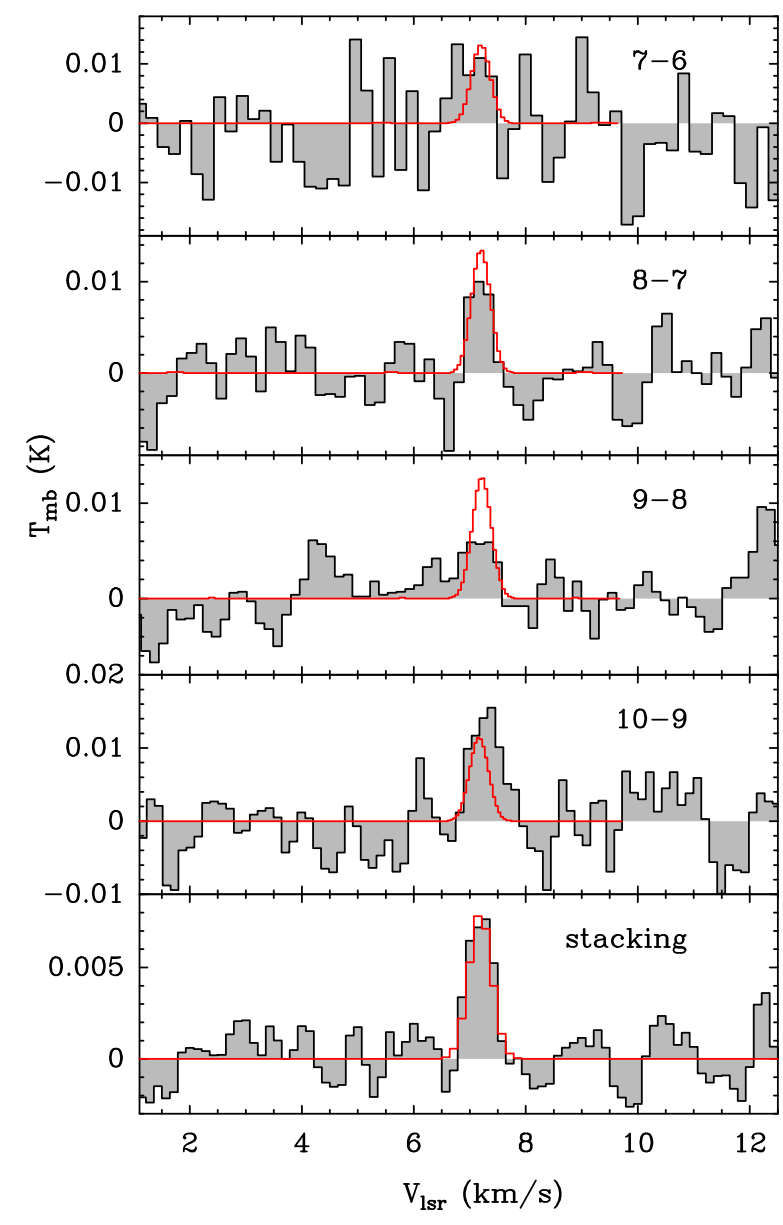

Fig. 1. Observed transitions of isocyanogen $(\mathrm{CNCN})$ in black with a LTE model (red) using $T_{\mathrm{ex}}=15 \mathrm{~K}$ and $N=6.5 \times 10^{11} \mathrm{~cm}^{-2}$. Bottom panel: results (in black) from the stacking of the four observed transitions as well as the Gaussian fit (in red).

result is shown in the bottom panel of Fig. 1 (in black, sampled to the resolution of the 10-9 transition) and shows an emission line (in red) detected at a $7 \sigma$ confidence over $12 \mathrm{~km} \mathrm{~s}^{-1}(7.7 \sigma$ over $5 \mathrm{~km} \mathrm{~s}^{-1}$ ), with the following parameters for the Gaussian fit: $T=8 \mathrm{mK}, F W H M=0.48 \mathrm{~km} \mathrm{~s}^{-1}, V_{\mathrm{LSR}}=7.19 \mathrm{~km} \mathrm{~s}^{-1}$. The CNCN detected transitions (8-7 and 10-9) present different line widths quoted in Table. $1\left(0.41\right.$ and $\left.0.61 \mathrm{~km} \mathrm{~s}^{-1}\right)$. These do, however, have high uncertainty on the $V_{\mathrm{LSR}}$ of the 8-7 transition. A similar difference, if confirmed, has been observed for the detection of CNCN in the L483 dense cloud (line widths between 0.47 and $0.87 \mathrm{~km} \mathrm{~s}^{-1}$ ) (Agúndez et al. 2018).

A feature seems to be present in the spectrum of the $9-8$ transition (Fig. 1) at about $12 \mathrm{~km} \mathrm{~s}^{-1}$. The unidentified (using the JPL and CDMS databases) transition is also seen in the spectrum of L483 (Agúndez et al. 2018) at $\sim 5 \mathrm{~km} \mathrm{~s}^{-1}$ with respect to the 9-8 transition. The selection of the baseline around $5 \mathrm{~km} \mathrm{~s}^{-1}$ is therefore necessary to avoid this feature, hence a $7.7 \sigma$ detection for the stacking (see above).

We will compute, in the following section, the column densities for the detected species and some upper limits.

\section{Determination of the column densities}

In this section we determine the column densities for the detected species $\left(\mathrm{CNCN}, \mathrm{NCCNH}^{+}, \mathrm{C}_{3} \mathrm{~N}, \mathrm{~A}-\mathrm{CH}_{3} \mathrm{CN}, \mathrm{E}-\mathrm{CH}_{3} \mathrm{CN}, \mathrm{H}_{2} \mathrm{CN}\right.$, $\mathrm{C}_{2} \mathrm{H}_{3} \mathrm{CN}$ ) and upper limits for $\mathrm{C}_{2} \mathrm{~N}$ and $\mathrm{C}_{4} \mathrm{~N}$, taking into account 
Table 1. Properties of the observed transitions for $E_{\mathrm{up}}$ lower than $30 \mathrm{~K}$.

\begin{tabular}{|c|c|c|c|c|c|c|c|c|c|}
\hline Species & QN & $\begin{array}{l}\text { Frequency } \\
(\mathrm{GHz})\end{array}$ & $\begin{array}{l}E_{\mathrm{up}} \\
(\mathrm{K})\end{array}$ & $\begin{array}{l}A_{i j} \\
\left(\mathrm{~s}^{-1}\right)\end{array}$ & $\begin{array}{l}\mathrm{rms} \\
(\mathrm{mK})\end{array}$ & $\begin{array}{c}T_{\mathrm{mb}} \\
(\mathrm{mK})\end{array}$ & $\begin{array}{c}W \\
\left(\mathrm{mK} \mathrm{km} \mathrm{s}^{-1}\right)\end{array}$ & $\begin{array}{l}F W H M \\
\left(\mathrm{~km} \mathrm{~s}^{-1}\right)\end{array}$ & $\begin{array}{c}V_{\mathrm{LSR}} \\
\left(\mathrm{km} \mathrm{s}^{-1}\right)\end{array}$ \\
\hline \multirow[t]{4}{*}{$\mathrm{CNCN}$} & $7-6$ & 72.436917 (multiplet) & 13.91 & $\geq 10^{-6}$ & 7.7 & - & - & - & - \\
\hline & $8-7$ & 82.784691 (multiplet) & 17.88 & $\geq 10^{-6}$ & 3.6 & $11.5 \pm 4.9$ & $5.0 \pm 3.8$ & $0.41 \pm 0.20$ & $7.2 \pm 0.1$ \\
\hline & $9-8$ & 93.1323 (multiplet) & 22.35 & $\geq 10^{-6}$ & 3.1 & - & - & - & - \\
\hline & $10-9$ & 103.4798 (multiplet) & 27.31 & $\geq 10^{-6}$ & 5.3 & $15.6 \pm 1.5$ & $10.1 \pm 2.1$ & $0.61 \pm 0.07$ & $7.3 \pm 0.1$ \\
\hline \multirow[t]{3}{*}{$\mathrm{NCCNH}^{+}$} & $9-8$ & 79.8826559 & 19.17 & $1.17 \times 10^{-4}$ & 5.3 & - & - & - & - \\
\hline & $10-9$ & 88.7581034 & 23.43 & $1.61 \times 10^{-4}$ & 2.4 & $16.6 \pm 2.2$ & $7.4 \pm 2.0$ & $0.42 \pm 0.06$ & $7.2 \pm 0.1$ \\
\hline & $11-10$ & 97.6334236 & 28.11 & $2.15 \times 10^{-4}$ & 5.4 & - & - & - & - \\
\hline \multirow[t]{6}{*}{$\mathrm{C}_{3} \mathrm{~N}$} & $8(J=17 / 2)-7(J=15 / 2)$ & 79.151 (triplet) & 17.09 & $\sim 2 \times 10^{-5}$ & 3.9 & $137.8 \pm 4.1$ & $64.3 \pm 4.8$ & $0.44 \pm 0.02$ & $7.3 \pm 0.1$ \\
\hline & $8(J=15 / 2)-7(J=13 / 2)$ & 79.1697 (triplet) & 17.10 & $\sim 2 \times 10^{-5}$ & 4.4 & $132.3 \pm 4.3$ & $57.5 \pm 4.7$ & $0.41 \pm 0.02$ & $7.2 \pm 0.1$ \\
\hline & $9(J=19 / 2)-8(J=17 / 2)$ & 89.0456 (triplet) & 21.37 & $\sim 3 \times 10^{-5}$ & 2.7 & $87.6 \pm 2.7$ & $35.3 \pm 2.0$ & $0.38 \pm 0.01$ & $7.3 \pm 0.1$ \\
\hline & $9(J=17 / 2)-8(J=15 / 2)$ & 89.0643 (triplet) & 21.37 & $\sim 3 \times 10^{-5}$ & 2.2 & $76.8 \pm 2.2$ & $28.5 \pm 2.4$ & $0.35 \pm 0.02$ & $7.3 \pm 0.1$ \\
\hline & $10(J=21 / 2)-9(J=19 / 2)$ & 98.9400 (triplet) & 26.11 & $\sim 4 \times 10^{-5}$ & 2.8 & $46.4 \pm 2.4$ & $18.2 \pm 1.9$ & $0.37 \pm 0.02$ & $7.3 \pm 0.1$ \\
\hline & $10(J=19 / 2)-9(J=17 / 2)$ & 98.9587 (triplet) & 26.12 & $\sim 4 \times 10^{-5}$ & 2.5 & $44.1 \pm 2.2$ & $16.8 \pm 1.8$ & $0.36 \pm 0.02$ & $7.3 \pm 0.1$ \\
\hline \multirow[t]{3}{*}{$\mathrm{A}-\mathrm{CH}_{3} \mathrm{CN}$} & $4_{0}-3_{0}$ & 73.5902185 & 8.83 & $3.17 \times 10^{-5}$ & 4.7 & $185.9 \pm 6.0$ & $122.2 \pm 7.9$ & $0.62 \pm 0.02$ & $7.3 \pm 0.1$ \\
\hline & $5_{0}-4_{0}$ & 91.9870877 & 13.24 & $6.33 \times 10^{-5}$ & 2.1 & $133.0 \pm 1.9$ & $66.3 \pm 2.35$ & $0.47 \pm 0.01$ & $7.3 \pm 0.1$ \\
\hline & $6_{0}-5_{0}$ & 110.3835001 & 18.54 & $1.11 \times 10^{-4}$ & 5.9 & $77.2 \pm 5.1$ & $29.5 \pm 4.4$ & $0.36 \pm 0.03$ & $7.3 \pm 0.1$ \\
\hline \multirow[t]{3}{*}{$\mathrm{E}-\mathrm{CH}_{3} \mathrm{CN}$} & $4_{1}-3_{1}$ & 73.5887995 & 7.95 & $2.97 \times 10^{-5}$ & 4.7 & $125.3 \pm 5.0$ & $77.0 \pm 9.7$ & $0.58 \pm 0.05$ & $7.2 \pm 0.1$ \\
\hline & $5_{1}-4_{1}$ & 91.9853142 & 12.36 & $6.07 \times 10^{-5}$ & 2.1 & $89.3 \pm 2.1$ & $49.2 \pm 2.1$ & $0.52 \pm 0.01$ & $7.3 \pm 0.1$ \\
\hline & $6_{1}-5_{1}$ & 110.3813721 & 17.66 & $1.08 \times 10^{-4}$ & 5.9 & $53.4 \pm 3.4$ & $27.7 \pm 4.0$ & $0.49 \pm 0.04$ & $7.3 \pm 0.1$ \\
\hline \multirow[t]{11}{*}{$\mathrm{C}_{2} \mathrm{H}_{3} \mathrm{CN}$} & $8_{0,8}-7_{0,7}$ & 75.5856915 & 16.35 & $3.44 \times 10^{-5}$ & 4.1 & $102.8 \pm 4.1$ & $41.6 \pm 3.8$ & $0.38 \pm 0.02$ & $7.3 \pm 0.1$ \\
\hline & $8_{1,8}-7_{1,7}$ & 73.9815530 & 18.15 & $3.18 \times 10^{-5}$ & 4.4 & $83.2 \pm 4.6$ & $31.0 \pm 3.5$ & $0.35 \pm 0.02$ & $7.3 \pm 0.1$ \\
\hline & $8_{1,7}-7_{1,6}$ & 77.6338240 & 18.94 & $3.67 \times 10^{-5}$ & 2.9 & $59.0 \pm 2.0$ & $28.3 \pm 2.8$ & $0.45 \pm 0.03$ & $7.3 \pm 0.1$ \\
\hline & $9_{0,9}-8_{0,8}$ & 84.9460000 & 20.43 & $4.92 \times 10^{-5}$ & 2.6 & $53.3 \pm 2.6$ & $28.4 \pm 3.1$ & $0.50 \pm 0.03$ & $7.3 \pm 0.1$ \\
\hline & $9_{1,9}-8_{1,8}$ & 83.2075051 & 22.14 & $4.56 \times 10^{-5}$ & 2.8 & $43.1 \pm 2.8$ & $21.1 \pm 2.7$ & $0.46 \pm 0.03$ & $7.3 \pm 0.1$ \\
\hline & $9_{1,8}-8_{1,7}$ & 87.3128120 & 23.13 & $5.27 \times 10^{-5}$ & 5.3 & $35.8 \pm 4.4$ & $19.4 \pm 5.0$ & $0.51 \pm 0.07$ & $7.2 \pm 0.1$ \\
\hline & $10_{0,10}-9_{0,9}$ & 94.2766360 & 24.95 & $6.76 \times 10^{-5}$ & 2.9 & $27.3 \pm 2.5$ & $12.8 \pm 2.6$ & $0.44 \pm 0.05$ & $7.3 \pm 0.1$ \\
\hline & $8_{2,7}-7_{2,6}$ & 75.8388620 & 25.04 & $3.26 \times 10^{-5}$ & 2.6 & $14.2 \pm 2.3$ & $7.7 \pm 2.8$ & $0.51 \pm 0.10$ & $7.2 \pm 0.1$ \\
\hline & $8_{2,6}-7_{2,5}$ & 76.1288810 & 25.08 & $3.30 \times 10^{-5}$ & 2.8 & $14.8 \pm 2.2$ & $7.1 \pm 3.4$ & $0.45 \pm 0.15$ & $7.3 \pm 0.1$ \\
\hline & $10_{1,10}-9_{1,9}$ & 92.4262500 & 26.58 & $6.30 \times 10^{-5}$ & 2.2 & $21.6 \pm 1.9$ & $10.1 \pm 1.8$ & $0.44 \pm 0.04$ & $7.3 \pm 0.1$ \\
\hline & $\begin{array}{c}\mathrm{N}_{K_{-1} K_{+1}}=1_{0,1}-0_{0,0} \\
\mathrm{~J} \mathrm{~F}_{1} \mathrm{~F}\end{array}$ & & & & & & & & \\
\hline \multirow[t]{6}{*}{$\mathrm{H}_{2} \mathrm{CN}$} & $1 / 23 / 25 / 2-1 / 23 / 25 / 2$ & 73.4442400 & 3.54 & $8.20 \times 10^{-6}$ & 3.6 & $19.1 \pm 3.8$ & $5.5 \pm 2.3$ & $0.27 \pm 0.06$ & $7.2 \pm 0.1$ \\
\hline & $3 / 21 / 23 / 2-1 / 21 / 23 / 2$ & 73.3693660 & 3.52 & $7.56 \times 10^{-6}$ & 4.2 & $14.0 \pm 3.9$ & $7.3 \pm 4.7$ & $0.49 \pm 0.18$ & $7.3 \pm 0.1$ \\
\hline & $3 / 25 / 23 / 2-1 / 23 / 21 / 2$ & 73.3425070 & 3.54 & $7.68 \times 10^{-6}$ & 4.1 & $14.4 \pm 3.9$ & $7.3 \pm 4.8$ & $0.48 \pm 0.19$ & $7.1 \pm 0.1$ \\
\hline & $3 / 25 / 25 / 2-1 / 23 / 23 / 2$ & 73.3454860 & 3.54 & $8.50 \times 10^{-6}$ & 4.0 & $17.4 \pm 4.1$ & $5.5 \pm 2.6$ & $0.30 \pm 0.07$ & $7.1 \pm 0.1$ \\
\hline & $3 / 21 / 21 / 2-1 / 2 \quad 1 / 23 / 2$ & 73.3492030 & 3.52 & $9.15 \times 10^{-6}$ & 4.1 & $7.9 \pm 3.9$ & $2.6 \pm 2.2$ & $0.51 \pm 0.20$ & $7.1 \pm 0.2$ \\
\hline & $3 / 15 / 27 / 2-1 / 23 / 25 / 2$ & 73.3496480 & 3.54 & $9.88 \times 10^{-6}$ & 4.1 & $26.6 \pm 3.1$ & $8.5 \pm 2.1$ & $0.30 \pm 0.04$ & $7.2 \pm 0.1$ \\
\hline
\end{tabular}

Notes. The spectroscopic parameters are from JPL for CNCN (Gerry et al. 1990) and CDMS (Müller et al. 2005) for NCCNH ${ }^{+}$(Amano \& Scappini 1991), $\mathrm{C}_{3} \mathrm{~N}$ (Gottlieb et al. 1983), $\mathrm{CH}_{3} \mathrm{CN}$ (Cazzoli \& Puzzarini 2006), $\mathrm{C}_{2} \mathrm{H}_{3} \mathrm{CN}$ (Müller et al. 2008) and $\mathrm{H}_{2} \mathrm{CN}$ (Yamamoto \& Saito 1992). The rms has been computed over a range of $15 \mathrm{~km} \mathrm{~s}^{-1}$ with a spectral resolution of $50 \mathrm{kHz}$.

the uncertainties based on the line fitting and also the absolute calibration accuracy, around $10 \%$ or better depending on the band considered. For that we used the CASSIS software, that interrogates the JPL and CDMS databases, and performs LTE and non LTE (when the collisions files are available) radiative transfer modelling. The number of detected transitions is not sufficient to explore a wide range of excitation temperatures.

For $\mathrm{NCCNH}^{+}$, we vary the excitation temperature between 10 and $15 \mathrm{~K}$, and compute a column density of $(1.2-1.9) \times$ $10^{10} \mathrm{~cm}^{-2}$. A lower value for the excitation temperature is not compatible with the upper limit on the 8-9 transition at $79.9 \mathrm{GHz}$. We note that the rms for both undetected transitions at 79.9 and $97.6 \mathrm{GHz}$ is more than a factor two higher than for the detected transition at $88.8 \mathrm{GHz}$, therefore the expected intensities (less than $20 \mathrm{mK}$ ) are within the noise level.
For $\mathrm{H}_{2} \mathrm{CN}$, we vary the excitation temperature between 5 and $15 \mathrm{~K}$ (a reasonable range for species detected in L1544), and compute a column density of $(3-6) \times 10^{11} \mathrm{~cm}^{-2}$. For isocyanogen $(\mathrm{CNCN})$, the covered energy range is large enough to constrain the excitation temperature between 13 and $17 \mathrm{~K}$, with a computed column density of $[6.0-6.6] \times 10^{11} \mathrm{~cm}^{-2}$.

For cyanoethynyl $\left(\mathrm{C}_{3} \mathrm{~N}\right)$, the resulting excitation temperature is $(7.2 \pm 0.2) \mathrm{K}$ and column density is $(1.6 \pm 0.1) \times 10^{12} \mathrm{~cm}^{-2}$. For $\mathrm{C}_{2} \mathrm{~N}$ and $\mathrm{C}_{4} \mathrm{~N}$, we can compute upper limits on the column densities of $2 \times 10^{12} \mathrm{~cm}^{-2}$ and $10^{14} \mathrm{~cm}^{-2}$ respectively.

For methyl cyanide $\left(\mathrm{CH}_{3} \mathrm{CN}\right)$, we used the $\mathrm{A}$ and $\mathrm{E}$ forms provided in the CASSIS database ${ }^{3}$ and converged on an excitation temperature of $5 \pm 0.5 \mathrm{~K}$ and a column density of $\left(4.5_{-1.4}^{+1.0}\right) \times 10^{11} \mathrm{~cm}^{-2}$ for the A form and $\left(2.8_{-0.9}^{+0.6}\right) \times 10^{11} \mathrm{~cm}^{-2}$

3 http://cassis.irap.omp.eu/?page=catalogs - vastel 


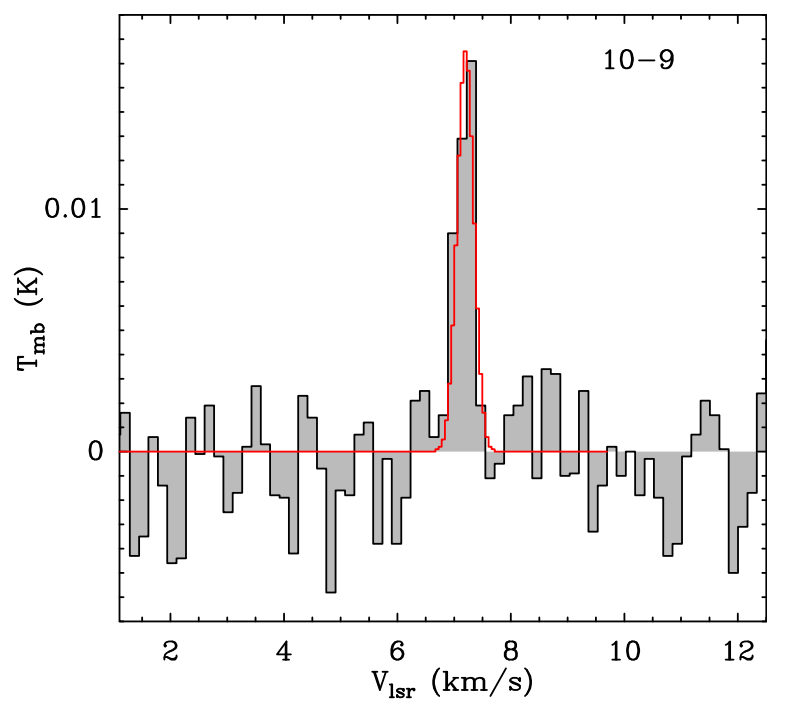

Fig. 2. Observed transition of protonated cyanogen $\left(\mathrm{NCCNH}^{+}\right)$with a LTE model (red) using $T_{\mathrm{ex}}=10 \mathrm{~K}$, and $N=1.9 \times 10^{10} \mathrm{~cm}^{-2}$.

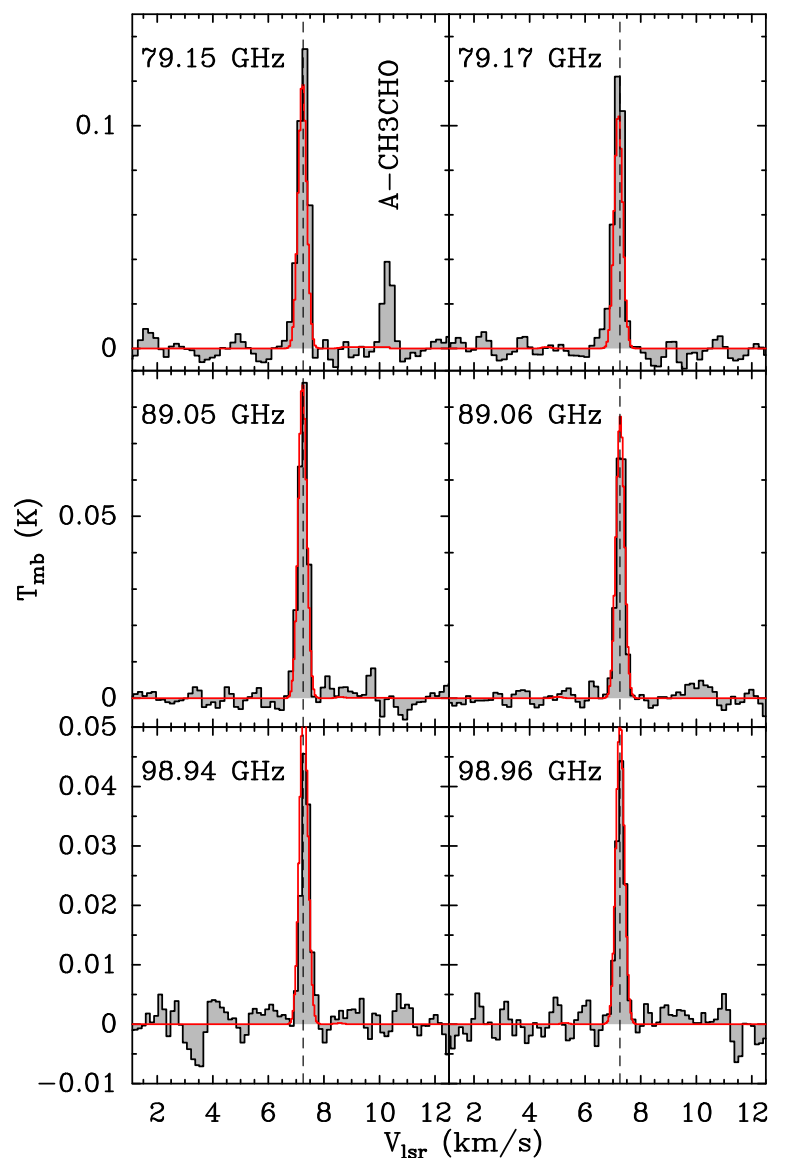

Fig. 3. Observed transitions of cyanoethynyl $\left(\mathrm{C}_{3} \mathrm{~N}\right)$ with an associated $V_{\mathrm{LSR}}$ of $7.25 \mathrm{~km} \mathrm{~s}^{-1}$ (dashed lines) with a LTE model (red) using $T_{\text {ex }}=7.2 \mathrm{~K}$ and $N=1.6 \times 10^{12} \mathrm{~cm}^{-2}$.

for the $\mathrm{E}$ form, with a $\mathrm{A} / \mathrm{E}$ ratio of $\sim 1.6$, slightly higher than the ratio found by Minh et al. (1993) towards TMC-1 $(\sim 1.5)$. For $\mathrm{C}_{2} \mathrm{H}_{3} \mathrm{CN}$, we detected 10 transitions between 16 and $27 \mathrm{~K}$, and used a rotational diagram analysis: $T_{\text {rot }}=(5.3 \pm 0.3) \mathrm{K}$ and $N=(4.0 \pm 0.5) \times 10^{12} \mathrm{~cm}^{-2}$. We note that for all species, we
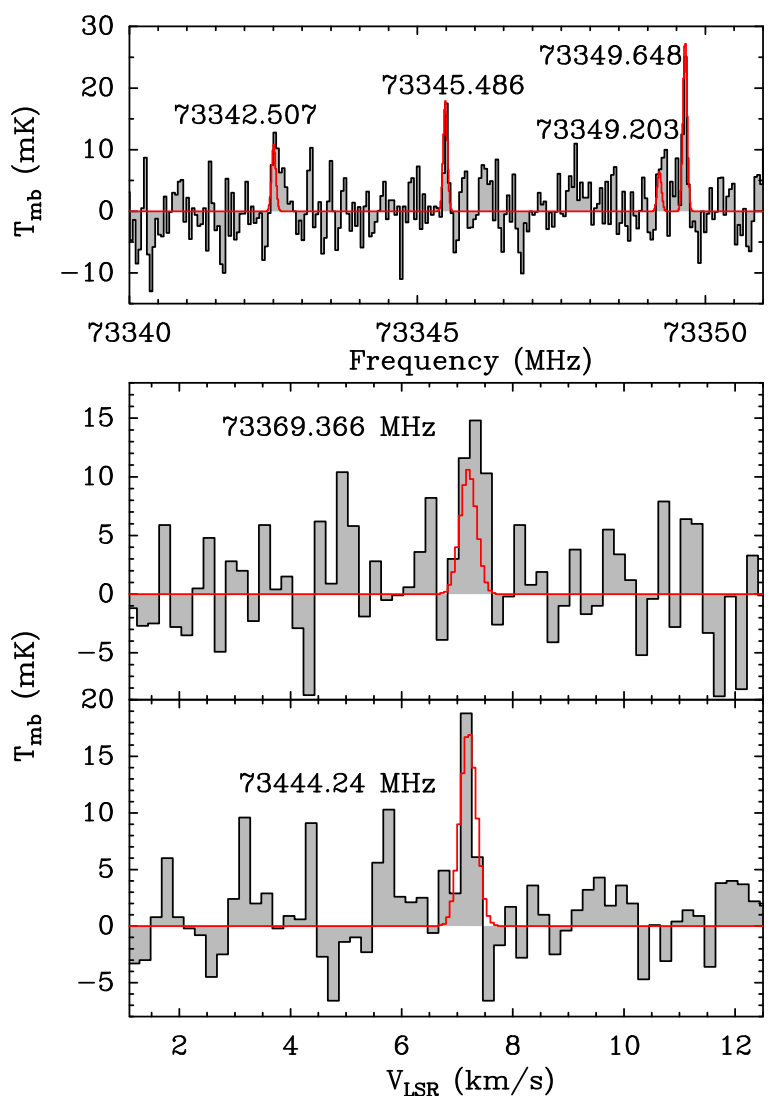

Fig. 4. Observed transitions of methylene amidogen $\left(\mathrm{H}_{2} \mathrm{CN}\right)$ with a LTE model (red): $T_{\mathrm{ex}}=10 \mathrm{~K}, N=3 \times 10^{11} \mathrm{~cm}^{-2}, \Delta V=0.35 \mathrm{~km} \mathrm{~s}^{-1}$.
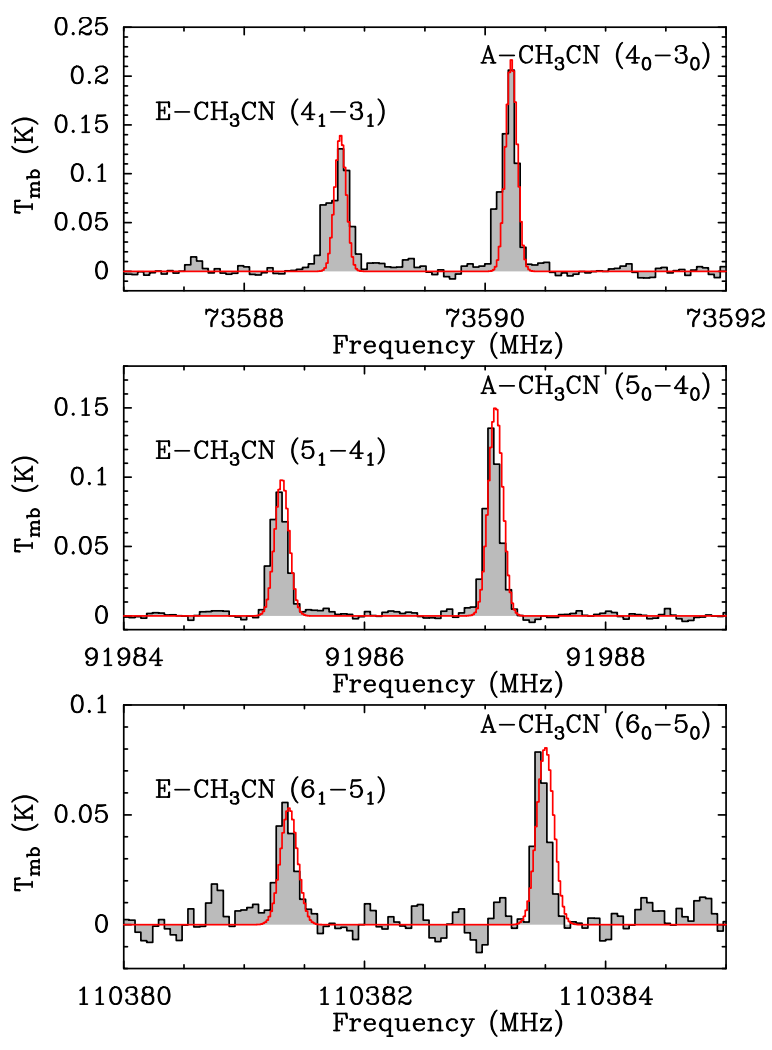

Fig. 5. Observed transitions of methyl cyanide $\left(\mathrm{CH}_{3} \mathrm{CN}\right)$ with a LTE model (red): $T_{\mathrm{ex}}=5 \mathrm{~K}, N(\mathrm{~A}$-form $)=4.5 \times 10^{11} \mathrm{~cm}^{-2}, N(\mathrm{E}-$ form $)=2.8 \times 10^{11} \mathrm{~cm}^{-2}, \Delta V=0.45 \mathrm{~km} \mathrm{~s}^{-1}$. 


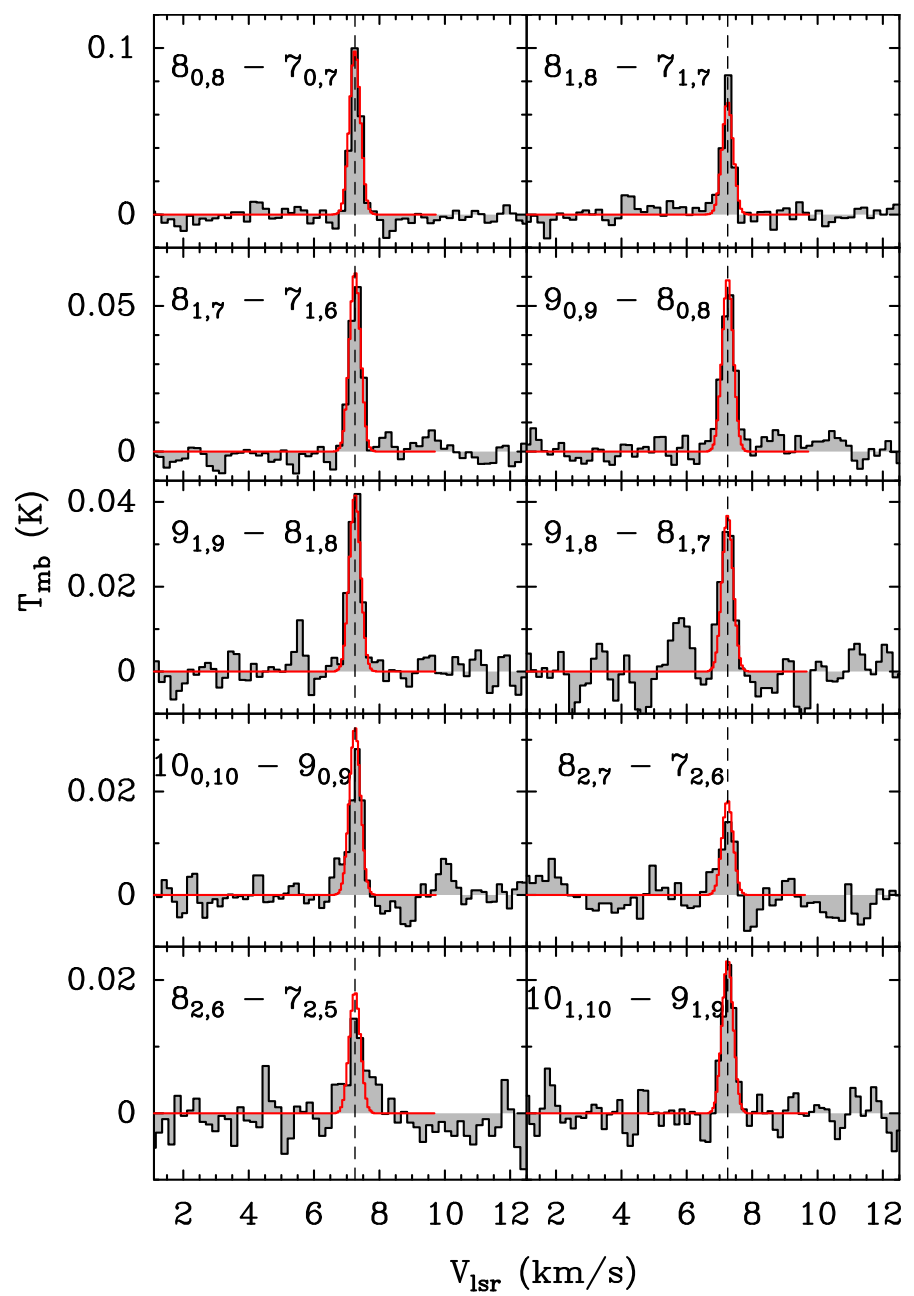

Fig. 6. Observed transitions of $\mathrm{C}_{2} \mathrm{H}_{3} \mathrm{CN}$ with an associated $V_{L S R}$ of $7.25 \mathrm{~km} \mathrm{~s}^{-1}$ (dashed lines) with a LTE model (red): $T_{\mathrm{ex}}=5.3 \mathrm{~K}$, $N=4.0 \times 10^{12} \mathrm{~cm}^{-2}, \Delta V=0.4 \mathrm{~km} \mathrm{~s}^{-1}$.

carefully checked that the modelled transitions of the undetected lines are consistent with the noise level.

We present in Table 2 a comparison between the observed column densities in the prototypical dark cloud TMC-1 and the values found in the L1544 prestellar core, at a later evolutionary stage. The latter values will be used in Sect. 4.2 for the chemical modelling of the core. The column densities are comparable for many species, such as $\mathrm{CN}, \mathrm{CNCN}, \mathrm{H}_{2} \mathrm{CN}, \mathrm{HCN}, \mathrm{HNC}, \mathrm{HCNH}^{+}$, despite the fact that TMC-1 is less evolved than L1544, which is showing signs of gravitational contraction in its centre (Caselli et al. 2012). For the other species, the column densities are higher in TMC-1, which can be explained by the fact that depletion is more pronounced in the prestellar core L1544.

\section{Chemical modelling}

\subsection{Investigating the chemistry of dicyanopolyynes species in the interstellar medium}

To build the chemical network for NCCN/CNCN/ $\mathrm{HC}_{2} \mathrm{~N}_{2}^{+}$chemistry we start from the work of Agúndez et al. $(2015,2018)$ using a general methodology for the study of the chemistry described in details in Loison et al. (2014, 2017). The rate constants used in our study are presented in Table 3 and the details of the calculations are shown in the appendix. They were added to the
Table 2. Comparison of the observed column densities (in $\mathrm{cm}^{-2}$ ) between the TMC-1 dark cloud and the L1544 prestellar core.

\begin{tabular}{|c|c|c|}
\hline & TMC-1 & L1544 \\
\hline \multirow[t]{2}{*}{$\mathrm{CN}$} & $(2.9 \pm 0.7) \times 10^{14}$ & $1.2 \times 10^{14}$ \\
\hline & Crutcher et al. (19 & (Hily-Blant et al. 2008) \\
\hline \multirow[t]{2}{*}{$\mathrm{C}_{3} \mathrm{~N}$} & $8.2 \times 10^{12}$ & $(1.6 \pm 0.1) \times 10^{12}$ \\
\hline & Guélin et al. (1998) & This paper \\
\hline \multirow[t]{2}{*}{ CNCN } & $9 \times 10^{11}$ & $(6.0-6.6) \times 10^{11}$ \\
\hline & Agúndez et al. (2018) & This paper \\
\hline \multirow[t]{2}{*}{$\mathrm{NCCNH}^{+}$} & $(8.6 \pm 4.4) \times 10^{10}$ & $(1.2-1.9) \times 10^{10}$ \\
\hline & Agúndez et al. (2015) & This paper \\
\hline \multirow{2}{*}{$\mathrm{H}_{2} \mathrm{CN}$} & $1.5 \times 10^{11}$ & $(3-6) \times 10^{11}$ \\
\hline & Ohishi et al. (1994) & This paper \\
\hline \multirow[t]{2}{*}{$\mathrm{CH}_{3} \mathrm{CN}$} & $5 \times 10^{12}$ & $(5-8.9) \times 10^{11}$ \\
\hline & Minh et al. (1993) & This paper \\
\hline \multirow[t]{2}{*}{$\mathrm{CH}_{2} \mathrm{CN}$} & $(2-10) \times$ & $(0.28-24.6) \times 10^{12}$ \\
\hline & Irvine et al. (1988) & Vastel et al. (2015b) \\
\hline \multirow[t]{2}{*}{$\mathrm{HCN}$} & $2.5 \times 10^{14}$ & $(2.7-4.4) \times 10^{14}$ \\
\hline & Hirota et al. (1998) & Quénard et al. (2017a) \\
\hline \multirow[t]{2}{*}{$\mathrm{HNC}$} & $4.2 \times 10^{14}$ & $(2-4) \times 10^{14}$ \\
\hline & Hirota et al. & Quénard et al. (2017a) \\
\hline \multirow[t]{3}{*}{$\mathrm{HC}_{3} \mathrm{~N}$} & $(1.6 \pm 0.1) \times$ & $(8.0 \pm 0.4) \times 10^{13}$ \\
\hline & Takano et al. (1998) & Quénard et al. (2017a) \\
\hline & & Hily-Blant et al. (2018) \\
\hline \multirow[t]{2}{*}{$\mathrm{HNC}_{3}$} & $(3.8 \pm 0.6) \times 10^{11}$ & $(0.75-2) \times 10^{11}$ \\
\hline & Kawaguchi et al. (1992a) & 1. $(2018 \mathrm{a})$ \\
\hline \multirow[t]{2}{*}{ HCCNC } & $(2.9 \pm 0.2) \times 10^{12}$ & $(0.85-2.2) \times 10^{12}$ \\
\hline & Kawaguchi et al. (1992b) & Vastel et al. (2018a) \\
\hline \multirow[t]{2}{*}{$\mathrm{HC}_{3} \mathrm{NH}^{+}$} & $(1.0 \pm 0.2) \times 10^{12}$ & $(1-2) \times 10^{11}$ \\
\hline & Kawaguchi et al. (1994) & Quénard et al. (2017a) \\
\hline \multirow[t]{2}{*}{$\mathrm{HCNH}^{+}$} & $1.9 \times 10^{13}$ & $(2 \pm 0.2) \times 10^{13}$ \\
\hline & Schilke et al. (1991) & Quénard et al. (2017a) \\
\hline \multirow[t]{2}{*}{$\mathrm{C}_{2} \mathrm{H}_{3} \mathrm{CN}$} & $3 \times 10^{13}$ & $(4.0 \pm 0.5) \times 10^{12}$ \\
\hline & Turner et al. (1999) & This paper \\
\hline $\mathrm{C}_{2} \mathrm{~N}$ & not detected & $\leq 2 \times 10^{12}$ \\
\hline $\mathrm{C}_{4} \mathrm{~N}$ & not detected & $\leq 10^{14}$ \\
\hline
\end{tabular}

chemical network of kida.uva.2014 (Wakelam et al. 2015) and additional updates from Wakelam et al. (2015); Loison et al. (2016); Hickson et al. (2016); Wakelam et al. (2017); Vidal et al. (2017); Loison et al. (2017).

The main NCCN production pathways in our network are the $\mathrm{CN}+\mathrm{HNC}$ and $\mathrm{N}+\mathrm{C}_{3} \mathrm{~N}$ reactions. A minor reaction is the $\mathrm{N}+\mathrm{HCCN}$ reaction which has been neglected in this study because HCCN is not supposed to be abundant in the interstellar medium. Indeed, HCCN has not been detected in TMC-1, with an upper limit on the fractional abundance (with respect to $\mathrm{H}_{2}$ ) of $2 \times 10^{-10}$ (McGonagle \& Irvine 1996), and it has not been included in our network. The $\mathrm{CN}+\mathrm{HNC}$ reaction has been studied theoretically by Petrie \& Osamura (2004) leading to a high rate constant at low temperature. The $\mathrm{N}+\mathrm{C}_{3} \mathrm{~N}$ reaction was already in the KIDA network but producing only $\mathrm{CN}+\mathrm{CCN}$ (Loison et al. 2014). For this study, we have performed an extensive theoretical calculations on the $\mathrm{C}_{3} \mathrm{~N}_{2}$ triplet surface showing that $\mathrm{N}\left({ }^{4} \mathrm{~S}\right)+\mathrm{C}_{3} \mathrm{~N}\left(\mathrm{X}^{2} \Sigma^{+}\right)$is correlated not only to $\mathrm{CN}\left(\mathrm{X}^{2} \Sigma^{+}\right)+$ $\mathrm{CCN}\left(\mathrm{X}^{2} \Sigma\right)$ but also to $\mathrm{C}\left({ }^{3} \mathrm{P}\right)+\mathrm{NCCN}\left(\mathrm{X}^{1} \Sigma^{+}\right)$and to $\mathrm{C}\left({ }^{3} \mathrm{P}\right)+$ $\mathrm{CNCN}\left(\mathrm{X}^{1} \Sigma^{+}\right)$as shown on Table A.1 and Figs. A.1, B.1, and C.1. Considering the exoenergicities, the $\mathrm{N}+\mathrm{C}_{3} \mathrm{~N} \rightarrow \mathrm{C}+\mathrm{NCCN}$ channel should be favoured. 
Table 3. Summary of reactions review.

\begin{tabular}{|c|c|c|c|c|c|c|c|}
\hline Reactions & $\begin{array}{c}\Delta E \\
\mathrm{~kJ} \mathrm{~mol}^{-1}\end{array}$ & $\alpha$ & $\beta$ & $\gamma$ & $F_{0}$ & $g$ & Reference \\
\hline $\begin{aligned} \mathrm{He}^{+}+\mathrm{C}_{2} \mathrm{~N}_{2} & \rightarrow \mathrm{CN}^{+}+\mathrm{CN}+\mathrm{He} \\
& \rightarrow \mathrm{CCN}^{+}+\mathrm{N}+\mathrm{He}\end{aligned}$ & $\begin{array}{l}-437 \\
-470\end{array}$ & $\begin{array}{l}8 \times 10^{-10} \\
8 \times 10^{-10}\end{array}$ & $\begin{array}{l}0 \\
0\end{array}$ & $\begin{array}{l}0 \\
0\end{array}$ & $\begin{array}{l}3 \\
3\end{array}$ & $\begin{array}{l}0 \\
0\end{array}$ & Rate constant from Raksit \& Bohme (1984) \\
\hline $\begin{aligned} \mathrm{He}^{+}+\mathrm{CNCN} & \rightarrow \mathrm{CN}^{+}+\mathrm{CN}+\mathrm{He} \\
& \rightarrow \mathrm{CNC}^{+}+\mathrm{N}+\mathrm{He}\end{aligned}$ & $\begin{array}{l}-522 \\
-695\end{array}$ & $\begin{array}{l}1.6 \times 10^{-10} \\
1.6 \times 10^{-10}\end{array}$ & $\begin{array}{l}-0.4 \\
-0.4\end{array}$ & $\begin{array}{l}0 \\
0\end{array}$ & $\begin{array}{l}3 \\
3\end{array}$ & $\begin{array}{l}0 \\
0\end{array}$ & $\begin{array}{l}\text { By comparison with } \mathrm{He}^{+}+\mathrm{NCCN} \text { taking into account the } \\
\text { dipolar moment of } \mathrm{CNCN}\end{array}$ \\
\hline $\mathrm{H}^{+}+\mathrm{C}_{2} \mathrm{~N}_{2} \rightarrow \mathrm{H}+\mathrm{C}_{2} \mathrm{~N}_{2}^{+}$ & -22 & $4.0 \times 10^{-9}$ & 0 & 0 & 3 & 0 & $\begin{array}{l}\text { Similar to } \mathrm{H}^{+}+\text {molecule without dipolar moment, } \\
\mathrm{IE}(\mathrm{NCCN})=13.37 \mathrm{eV}\end{array}$ \\
\hline $\begin{aligned} \mathrm{H}^{+}+\mathrm{CNCN} & \rightarrow \mathrm{H}+\mathrm{CNCN}^{+} \\
& \rightarrow \mathrm{H}+\mathrm{C}_{2} \mathrm{~N}_{2}^{+} \\
& \rightarrow \mathrm{NCN}^{+}+\mathrm{CH} \\
& \rightarrow \mathrm{CH}^{+}+\mathrm{NCN}\end{aligned}$ & $\begin{array}{r}-65 \\
-99 \\
+380 \\
+154\end{array}$ & $\begin{array}{c}0 \\
1.0 \times 10^{-8} \\
0 \\
0\end{array}$ & -0.4 & 0 & 3 & 0 & $\begin{array}{l}\text { Similar to } \mathrm{H}^{+}+\text {molecule with dipolar moment } \\
\text { We avoid introducing } \mathrm{CNCN}+\text { which will react quickly } \\
\text { with } \mathrm{H}_{2} \text { leading to the same final products that } \mathrm{C}_{2} \mathrm{~N}_{2}^{+}\end{array}$ \\
\hline $\begin{aligned} \mathrm{H}+\mathrm{NC}_{3} \mathrm{~N} & \rightarrow \mathrm{HCN}+\mathrm{C}_{2} \mathrm{~N} \\
& \rightarrow \mathrm{HNC}+\mathrm{C}_{2} \mathrm{~N} \\
& \rightarrow \mathrm{CN}+\mathrm{HCCN}\end{aligned}$ & $\begin{array}{c}+15 \\
+67 \\
+108\end{array}$ & $\begin{array}{l}0 \\
0 \\
0\end{array}$ & & & & & $\begin{array}{l}\mathrm{NC}_{3} \mathrm{~N} \text { has a triplet ground state so it will react quickly } \\
\text { with atoms and radicals. The two unpaired electrons are } \\
\text { localized on central } \mathrm{C} \text { atom and on terminal } \mathrm{N} \text { atoms: } \\
\mathrm{N} \equiv \mathrm{C}--^{\bullet} \mathrm{C}=\mathrm{C}=\mathrm{N}^{\bullet} \leftrightarrow \leftrightarrow^{\bullet} \mathrm{N}=\mathrm{C}=\bullet^{\bullet} \mathrm{C}-\mathrm{C} \equiv \mathrm{N} \\
\text { The } \bullet \text { represents the lonely electron and show the reactive sites }\end{array}$ \\
\hline $\begin{aligned} & \mathrm{C}^{+}+\mathrm{C}_{2} \mathrm{~N}_{2} \rightarrow \mathrm{CNC}^{+}+\mathrm{CN} \\
& \rightarrow \mathrm{CCN}^{+}+\mathrm{CN} \\
&\end{aligned}$ & $\begin{array}{r}-65 \\
+77 \\
\end{array}$ & $\begin{array}{c}1.0 \times 10^{-9} \\
0\end{array}$ & 0 & 0 & 3 & 0 & $\begin{array}{l}\text { Charge exchange is endothermic. Rate constant similar } \\
\text { to } \mathrm{C}^{+}+\mathrm{C}_{2} \mathrm{H}_{2} \text {, close to capture rate }\end{array}$ \\
\hline $\begin{aligned} \mathrm{C}^{+}+\mathrm{CNCN} & \rightarrow \mathrm{CNC}^{+}+\mathrm{CN} \\
& \rightarrow \mathrm{CCN}^{+}+\mathrm{CN}\end{aligned}$ & $\begin{array}{c}-149 \\
-8\end{array}$ & $\begin{array}{c}2.0 \times 10^{-9} \\
0\end{array}$ & -0.4 & 0 & 3 & 0 & $\begin{array}{l}\text { Charge exchange is endothermic. Rate constant similar to } \\
\mathrm{C}^{+}+\mathrm{HCN} \text {, close to capture rate with temperature } \\
\text { dependency from charge-dipole interaction }\end{array}$ \\
\hline $\begin{aligned} \mathrm{C}+\mathrm{C}_{2} \mathrm{~N}_{2} & \rightarrow \mathrm{NC}_{3} \mathrm{~N}+\mathrm{h} v \\
& \rightarrow \mathrm{C}_{3}+\mathrm{N}_{2} \\
& \rightarrow \mathrm{CN}+\mathrm{CCN} \\
& \rightarrow \mathrm{N}+\mathrm{C}_{3} \mathrm{~N} \\
& \rightarrow \mathrm{C}_{2}+\mathrm{NCN}\end{aligned}$ & $\begin{array}{l}-435 \\
-294 \\
+98 \\
+157 \\
+266\end{array}$ & $\begin{array}{c}2.0 \times 10^{-14} \\
0 \\
0 \\
0 \\
0\end{array}$ & -1.5 & 0 & 10 & 0 & $\begin{array}{l}\text { The results from Safrany \& Jaster (1968b,a) and Whyte \& Phillips (1983) } \\
\text { are complex, the products assumed for this reaction, } \mathrm{CN}+\mathrm{CCN} \text {, being } \\
\text { widely endothermic. We have performed theoretical calculations } \\
\text { (see appendix). We propose that the experimental observations are } \\
\text { due to 3-body } \mathrm{NC}_{3} \mathrm{~N} \text { formation. In interstellar molecular clouds } \\
\text { the pressure is too low for 3-body reactions but radiative association } \\
\text { may play a role. The radiative association rate constant is } \\
\text { calculated using our } \rho \text {-association model (Hebrard et al. 2013) }\end{array}$ \\
\hline $\mathrm{C}+\mathrm{CNCN} \rightarrow \mathrm{C}+\mathrm{C}_{2} \mathrm{~N}_{2}$ & -84 & $1.0 \times 10^{-10}$ & 0 & 0 & 3 & 0 & See text and appendix \\
\hline $\mathrm{C}+\mathrm{NC}_{3} \mathrm{~N} \rightarrow \mathrm{CN}+\mathrm{C}_{3} \mathrm{~N}$ & -142 & $2.0 \times 10^{-10}$ & 0 & 0 & 3 & 0 & 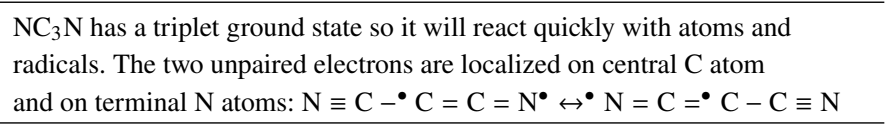 \\
\hline $\begin{aligned} & \mathrm{CH}+\mathrm{C}_{2} \mathrm{~N}_{2} \rightarrow \mathrm{C}_{2} \mathrm{~N}+\mathrm{HCN} \\
& \rightarrow \mathrm{HCCN}+\mathrm{CN} \\
& \rightarrow \mathrm{C}_{3} \mathrm{H}+\mathrm{N}_{2} \\
&\end{aligned}$ & $\begin{array}{l}-81 \\
+20 \\
-177 \\
\end{array}$ & $\begin{array}{c}2.0 \times 10^{-10} \\
0 \\
0\end{array}$ & $\begin{array}{l}0 \\
0 \\
0\end{array}$ & $\begin{array}{l}0 \\
0 \\
0\end{array}$ & $\begin{array}{l}3 \\
0 \\
0\end{array}$ & $\begin{array}{l}0 \\
0 \\
0\end{array}$ & $\begin{array}{l}\text { There is very likely no barrier for this reaction considering the very high } \\
\text { reactivity of the } \mathrm{CH} \text { radical }\end{array}$ \\
\hline $\mathrm{CN}+\mathrm{HNC} \rightarrow \mathrm{C}_{2} \mathrm{~N}_{2}+\mathrm{H}$ & -103 & $2.0 \times 10^{-10}$ & 0 & 0 & 4 & 0 & See Petrie \& Osamura (2004) \\
\hline $\mathrm{CN}+\mathrm{HCNH}^{+} \rightarrow \mathrm{HC}_{2} \mathrm{~N}_{2}^{+}+\mathrm{H}$ & +11 & "0" & & & & & $\begin{array}{l}\text { The reaction is endothermic and show a barrier of } 52 \mathrm{~kJ} \mathrm{~mol}^{-1} \\
\text { at M06-2X/AVTZ level (see Table G.1) }\end{array}$ \\
\hline $\mathrm{N}+\mathrm{C}_{2} \mathrm{~N}_{2} \rightarrow \mathrm{C}_{2} \mathrm{~N}_{2}+\mathrm{H}$ & & "0" & & & & & $\begin{array}{l}\text { Very large barriers for } \mathrm{N}^{-1} \text { addition on the nitrogen }\left(91 \mathrm{~kJ} \mathrm{~mol}^{-1}\right) \text { or on the } \\
\text { carbon atoms }\left(63 \mathrm{~kJ} \mathrm{~mol}^{-1}\right) \text { (M06-2X/AVTZ calculations). The rate } \\
\text { constant value in Safrany \& Jaster (1968c) is likely due to the presence } \\
\text { of } \mathrm{N}_{2}^{*} \text { (Michael 1980; Dutuit et al. 2013) }\end{array}$ \\
\hline $\mathrm{N}+\mathrm{CNCN} \rightarrow \mathrm{C}_{2} \mathrm{~N}_{2}+\mathrm{H}$ & & "0" & & & & & $\begin{array}{l}\text { The most favourable attack on final carbon atom shows a large barrier for } \mathrm{N} \\
\text { addition }\left(32 \mathrm{~kJ} \mathrm{~mol}^{-1}\right)(\mathrm{M} 06-2 \mathrm{X} / \mathrm{AVTZ} \text { calculations })\end{array}$ \\
\hline $\begin{aligned} \mathrm{N}+\mathrm{C}_{3} \mathrm{~N} & \rightarrow \mathrm{C}+\mathrm{C}_{2} \mathrm{~N}_{2} \\
& \rightarrow \mathrm{C}+\mathrm{CNCN} \\
& \rightarrow \mathrm{CN}+\mathrm{C}_{2} \mathrm{~N} \\
& \rightarrow \mathrm{C}_{3}+\mathrm{N}_{2}\end{aligned}$ & $\begin{array}{l}-157 \\
-72 \\
-43 \\
-333\end{array}$ & $\begin{array}{l}6.0 \times 10^{-11} \\
1.0 \times 10^{-11} \\
2.0 \times 10^{-11} \\
\quad 0\end{array}$ & $\begin{array}{l}0 \\
0 \\
0 \\
0\end{array}$ & $\begin{array}{l}0 \\
0 \\
0 \\
0\end{array}$ & $\begin{array}{l}3 \\
3 \\
3 \\
3\end{array}$ & $\begin{array}{l}0 \\
0 \\
0 \\
0\end{array}$ & $\begin{array}{l}\text { Theoretical calculations. Important NCCN formation pathway } \\
\text { in dense molecular clouds }\end{array}$ \\
\hline $\begin{aligned} \mathrm{N}+\mathrm{NC}_{3} \mathrm{~N} & \rightarrow \mathrm{CN}+\mathrm{C}_{2} \mathrm{~N}_{2} \\
& \rightarrow \mathrm{N}_{2}+\mathrm{C}_{3} \mathrm{~N}\end{aligned}$ & $\begin{array}{l}-299 \\
-337\end{array}$ & $\begin{array}{l}4.0 \times 10^{-11} \\
4.0 \times 10^{-11}\end{array}$ & $\begin{array}{l}0 \\
0\end{array}$ & $\begin{array}{l}0 \\
0\end{array}$ & $\begin{array}{l}3 \\
3\end{array}$ & $\begin{array}{l}0 \\
0\end{array}$ & $\begin{array}{l}\mathrm{NC}_{3} \mathrm{~N} \text { has a triplet ground state so it will react quickly with atoms and radicals } \\
\text { The two unpaired electrons are localized on central } \mathrm{C} \text { atom and on } \\
\text { terminal } \mathrm{N} \text { atomes } \mathrm{N} \equiv \mathrm{C}-\bullet^{\bullet} \mathrm{C}=\mathrm{C}=\mathrm{N}^{\bullet} \leftrightarrow \leftrightarrow^{\bullet} \mathrm{N}=\mathrm{C}==^{\bullet} \mathrm{C}-\mathrm{C} \equiv \mathrm{N}\end{array}$ \\
\hline $\mathrm{C}_{2} \mathrm{~N}_{2}^{+}+\mathrm{H}_{3}^{+} \rightarrow \mathrm{HC}_{2} \mathrm{~N}_{2}^{+}+\mathrm{H}_{2}$ & -215 & $2.8 \times 10^{-9}$ & 0 & 0 & 1.2 & 0 & See Anicich (2003). \\
\hline $\mathrm{C}_{2} \mathrm{~N}_{2}^{+}+\mathrm{HCO}^{+} \rightarrow \mathrm{HC}_{2} \mathrm{~N}_{2}^{+}+\mathrm{CO}$ & -67 & $1.7 \times 10^{-9}$ & 0 & 0 & 2 & 0 & $\begin{array}{l}\text { By comparison with } \mathrm{NCCN}+\mathrm{H}_{3}^{+} \text {considering the } \\
\mathrm{H}_{3}^{+} / \mathrm{HCO}^{+} \text {masses difference }\end{array}$ \\
\hline
\end{tabular}

Notes. Definition of $\alpha, \beta, \gamma, F_{0}, g$ can been found in Wakelam et al. $(2012,2010): k=\alpha \times(T / 300)^{\beta} \times \exp (-\gamma / T) \mathrm{cm}^{3}$ molecule $\mathrm{s}^{-1}, T$ is the temperature and ranges between 10 and $300 \mathrm{~K}$ except for some cases (noted).

A91, page 6 of 19 
Table 3. continued.

\begin{tabular}{|c|c|c|c|c|c|c|c|}
\hline Reactions & $\begin{array}{c}\Delta E \\
\mathrm{~kJ} \mathrm{~mol}^{-1}\end{array}$ & $\alpha$ & $\beta$ & $\gamma$ & $F_{0}$ & $g$ & Reference \\
\hline $\begin{aligned} \mathrm{CNCN}+\mathrm{H}_{3}^{+} & \rightarrow \mathrm{HCNCN}^{+}+\mathrm{H}_{2} \\
& \rightarrow \mathrm{HNCNC}^{+}+\mathrm{H}_{2} \\
& \rightarrow \mathrm{HC}_{2} \mathrm{~N}_{2}++\mathrm{H}_{2}\end{aligned}$ & $\begin{array}{l}-240 \\
-256\end{array}$ & $\begin{array}{c}0 \\
0 \\
2.8 \times 10^{-9}\end{array}$ & -0.3 & 0 & 2 & 0 & $\begin{array}{l}\text { By comparison with } \mathrm{NCCN}+\mathrm{H}_{3}^{+} \text {taking into account the low dipole moment } \\
\text { of } \mathrm{CNCN} \text {. We do not introduce } \mathrm{HC}_{2} \mathrm{~N}_{2}^{+} \text {isomers as their DR will leads to } \\
\text { similar products with our statistical calculations }\end{array}$ \\
\hline $\begin{aligned} \mathrm{CNCN}+\mathrm{HCO}^{+} & \rightarrow \mathrm{HCNCN}^{+}+\mathrm{CO} \\
& \rightarrow \mathrm{HNCNC}^{+}+\mathrm{CO} \\
& \rightarrow \mathrm{HC}_{2} \mathrm{~N}_{2}^{+}+\mathrm{CO}\end{aligned}$ & $\begin{array}{l}-92 \\
-109\end{array}$ & $\begin{array}{c}0 \\
0 \\
1.6 \times 10^{-9}\end{array}$ & -0.3 & 0 & 2 & 0 & $\begin{array}{l}\text { We do not introduce } \mathrm{HC}_{2} \mathrm{~N}_{2}^{+} \text {isomers as their } \mathrm{DR} \text { will leads to similar products } \\
\text { with our statistical calculations }\end{array}$ \\
\hline $\begin{aligned} & \mathrm{C}_{2} \mathrm{~N}_{2}^{+}+\mathrm{H}_{2} \rightarrow \mathrm{HNCCN}^{+}+\mathrm{H} \\
& \rightarrow \mathrm{HC}_{2} \mathrm{~N}_{2}^{+}+\mathrm{H} \\
&\end{aligned}$ & -200 & $\begin{array}{c}0 \\
9.0 \times 10^{-10} \\
\end{array}$ & 0 & 0 & 1.4 & 0 & Anicich (2003) \\
\hline $\begin{aligned} \mathrm{CNCN}^{+}+\mathrm{H}_{2} & \rightarrow \mathrm{HNCNC}^{+}+\mathrm{H} \\
& \rightarrow \mathrm{HC}_{2} \mathrm{~N}_{2}^{+}+\mathrm{H}\end{aligned}$ & -190 & $\begin{array}{c}0 \\
9.0 \times 10^{-10}\end{array}$ & 0 & 0 & 2 & 0 & $\begin{array}{l}\text { Same as } \mathrm{C}_{2} \mathrm{~N}_{2}^{+}+\mathrm{H}_{2} \text {. We do not introduce } \mathrm{HC}_{2} \mathrm{~N}_{2}^{+} \text {isomers } \\
\text { as their DR will leads to similar products with our statistical calculations }\end{array}$ \\
\hline $\begin{aligned} \mathrm{HC}_{2} \mathrm{~N}_{2}^{+}+\mathrm{e}^{-} & \rightarrow \mathrm{H}+\mathrm{C}_{2} \mathrm{~N}_{2} \\
& \rightarrow \mathrm{H}+\mathrm{CNCN} \\
& \rightarrow \mathrm{HCN}+\mathrm{CN} \\
& \rightarrow \mathrm{HNC}+\mathrm{CN} \\
& \rightarrow \mathrm{H}+\mathrm{CN}+\mathrm{CN}\end{aligned}$ & $\begin{array}{l}-668 \\
-583 \\
-616 \\
-564 \\
-93\end{array}$ & $\begin{array}{c}1.7 \times 10^{-7} \\
1.0 \times 10^{-8} \\
1.0 \times 10^{-7} \\
1.0 \times 10^{-7} \\
0\end{array}$ & & & & & $\begin{array}{l}\mathrm{NCCN} / \mathrm{CNCN} \text { branching calculated using statistical theory, as well } \\
\text { as } \mathrm{HNC} / \mathrm{HCN} \text { ratio. }\end{array}$ \\
\hline $\begin{aligned} \mathrm{HC}_{3} \mathrm{NH}^{+}+\mathrm{e}^{-} & \rightarrow \mathrm{HC}_{3} \mathrm{~N}+\mathrm{H} \\
& \rightarrow \mathrm{HNC}_{3}+\mathrm{H} \\
& \rightarrow \mathrm{H}+\mathrm{HCNCC} \\
& \rightarrow \mathrm{H}+\mathrm{HCCNC} \\
& \rightarrow \mathrm{C}_{2} \mathrm{H}+\mathrm{HCN} \\
& \rightarrow \mathrm{C}_{2} \mathrm{H}+\mathrm{HNC} \\
& \rightarrow \mathrm{C}_{3} \mathrm{~N}+\mathrm{H}+\mathrm{H}\end{aligned}$ & $\begin{array}{r}-568 \\
-369 \\
-250 \\
-473 \\
-377 \\
-345 \\
+29\end{array}$ & $\begin{array}{c}6.0 \times 10^{-7} \\
1.4 \times 10^{-7} \\
1.0 \times 10^{-8} \\
3.0 \times 10^{-8} \\
3.6 \times 10^{-7} \\
3.6 \times 10^{-7} \\
0\end{array}$ & $\begin{array}{l}-0.58 \\
-0.58 \\
-0.58 \\
-0.58 \\
-0.58 \\
-0.58 \\
-0.58\end{array}$ & $\begin{array}{l}0 \\
0 \\
0 \\
0 \\
0 \\
0 \\
0\end{array}$ & $\begin{array}{l}3 \\
3 \\
3 \\
3 \\
3 \\
3 \\
3\end{array}$ & $\begin{array}{l}0 \\
0 \\
0 \\
0 \\
0 \\
0 \\
0\end{array}$ & $\begin{array}{l}\text { The global rate constant and the } 52 \% \text { of } \mathrm{HC}_{3} \mathrm{~N} \text { isomers formation and } \\
48 \% \text { of } \mathrm{HCN} \text { isomers formation is from } \\
\text { Geppert et al. (2004); Vigren et al. (2012) using also Osamura et al. (1999) } \\
\text { The relative } \mathrm{HC}_{3} \mathrm{~N} / \mathrm{HNC}_{3} / \mathrm{HCNCC} / \mathrm{HCCNC} \text { and } \mathrm{HCN} / \mathrm{HNC} \\
\text { branching ratios are arbitrary }\end{array}$ \\
\hline
\end{tabular}

Apart from the minor channel $\mathrm{N}+\mathrm{C}_{3} \mathrm{~N} \rightarrow \mathrm{C}+\mathrm{CNCN}$, the other $\mathrm{CNCN}$ production is through the electronic dissociation rate (DR) of $\mathrm{HC}_{2} \mathrm{~N}_{2}^{+}$, the latter being produced through $\mathrm{NCCN}+\mathrm{H}_{3}^{+}$and $\mathrm{NCCN}+\mathrm{HCO}^{+}$reactions (see Eq. (3)) Other $\mathrm{HC}_{2} \mathrm{~N}_{2}^{+}$production pathways show a barrier as $\mathrm{CN}+$ $\mathrm{HCNH}^{+}$(see Table G.1). By comparison with similar DRs (Florescu-Mitchell \& Mitchell 2006; Plessis et al. 2012; Reiter \& Janev 2010), the H elimination seems to be an important pathway, assumed to be equal to $50 \%$ in this study. In that case, the NCCN production is highly exothermic and will carry a large internal energy, up to $668 \mathrm{~kJ} \mathrm{~mol}^{-1}$ (see Table 3). If the internal energy is above the dissociation limit, it will lead to dissociation producing $\mathrm{H}+\mathrm{CN}+\mathrm{CN}$ with a low branching ratio neglected here. On the contrary, if the internal energy is below the dissociation limit but above the isomerization barrier which is the most probable case as hydrogen atom and NCCN will cary some translational energy, it will lead to the production of NCCN and $\mathrm{CNCN}$ isomers as shown on Fig. D.1 and Table D.1. The branching ratio is assumed to be proportional to the ro-vibrational density of states of each isomer at the transition state (TS) energy (Herbst et al. 2000). As the DR of $\mathrm{HC}_{2} \mathrm{~N}_{2}^{+}$and $\mathrm{CNCNH}^{+}$will lead to very similar products $(\mathrm{NCCN}, \mathrm{CNCN}, \mathrm{HCN}, \mathrm{HNC}$, and $\mathrm{CN}$ ) with very similar branching ratios for our assumptions, we consider only one isomer for $\mathrm{HC}_{2} \mathrm{~N}_{2}^{+}$and $\mathrm{CNCNH}^{+}$.

Ionic reactions are efficient $\mathrm{NCCN}$ and $\mathrm{CNCN}$ destruction pathways, either directly through reaction with $\mathrm{He}^{+}$ (Raksit \& Bohme 1984) or through protonation as the DR of $\mathrm{HC}_{2} \mathrm{~N}_{2}^{+}$does not lead to $100 \%$ of $\mathrm{NCCN}$ and CNCN. Safrany \& Jaster (1968a) have found some reactivity of NCCN with nitrogen atoms. However, it has been shown that, in this kind of experiment, the reactivity is due to the presence of metastable $\mathrm{N}_{2}^{*}$ (Michael 1980; Dutuit et al. 2013). We then performed DFT calculations, using the M06-2X/AVTZ method (this highly nonlocal functional developed by Zhao \& Truhlar (2008) is well suited for structures and energetics of the transition states). Our calculations show that the $\mathrm{N}+\mathrm{NCCN}$ and $\mathrm{N}+$ $\mathrm{CNCN}$ reactions have very large barriers in the entrance valley as shown on Tables E.1 and F.1 and are negligible at low temperatures.

We have also performed calculations on the $\mathrm{N}$ and $\mathrm{O}$ reactions with $\mathrm{CNCN}$ and the $\mathrm{H}, \mathrm{N}, \mathrm{O}$ reactions with $\mathrm{NCCN}$, see Tables E.1 and F.1 showing in all cases large barrier in the entrance valley and then negligible rates at low temperatures. Safrany \& Jaster (1968b) and Whyte \& Phillips (1983) deduced from a complex experiment that carbon atoms quickly react with NCCN at room temperature, with a rate constant equal to $3 \times 10^{-11} \mathrm{~cm}^{3}$ molecules ${ }^{-1} \mathrm{~s}^{-1}$. Their experiments are complex but are reliable on the fact that carbon atoms react with $\mathrm{NCCN}$, leading directly or indirectly to $\mathrm{CN}$ radicals. However, the products assumed for this reaction, $\mathrm{CN}+\mathrm{CCN}$, are widely endothermic as shown of Fig. A.1. The only exothermic exit channel for this reaction, $\mathrm{C}_{3}+\mathrm{N}_{2}$, is spin forbidden. Moreover, $\mathrm{C}_{3}$ and $\mathrm{N}_{2}$ cannot be the only products of the experiments since $\mathrm{C}_{3}$ and $\mathrm{N}_{2}$ have a very low reactivity towards nitrogen atoms contrary to the product obtained in the experiments. They cannot lead to $\mathrm{CN}$ which was identified as a product of the $\mathrm{C}+\mathrm{NCCN}$ reaction in presence of $\mathrm{N}$ atoms.

An alternative explanation is the formation of NCCCN. In the experiment at few Torr, some, or most, NCCCN adduct will be stabilized through collisions and will react with the nitrogen atoms to produce $\mathrm{CN}$ and $\mathrm{C}_{3} \mathrm{~N}$, as $\mathrm{NCCCN}$ has a triplet ground state and is reactive with atoms and radicals. In interstellar molecular clouds the pressure is too low for three-body reactions but radiative association may play a role.

Using a model developed in Hebrard et al. (2013), we calculated that $k(T)=2 \times 10^{-14} \times(T / 300)^{-1.5} \mathrm{~cm}^{3}$ molecules ${ }^{-1} \mathrm{~s}^{-1}$ for 

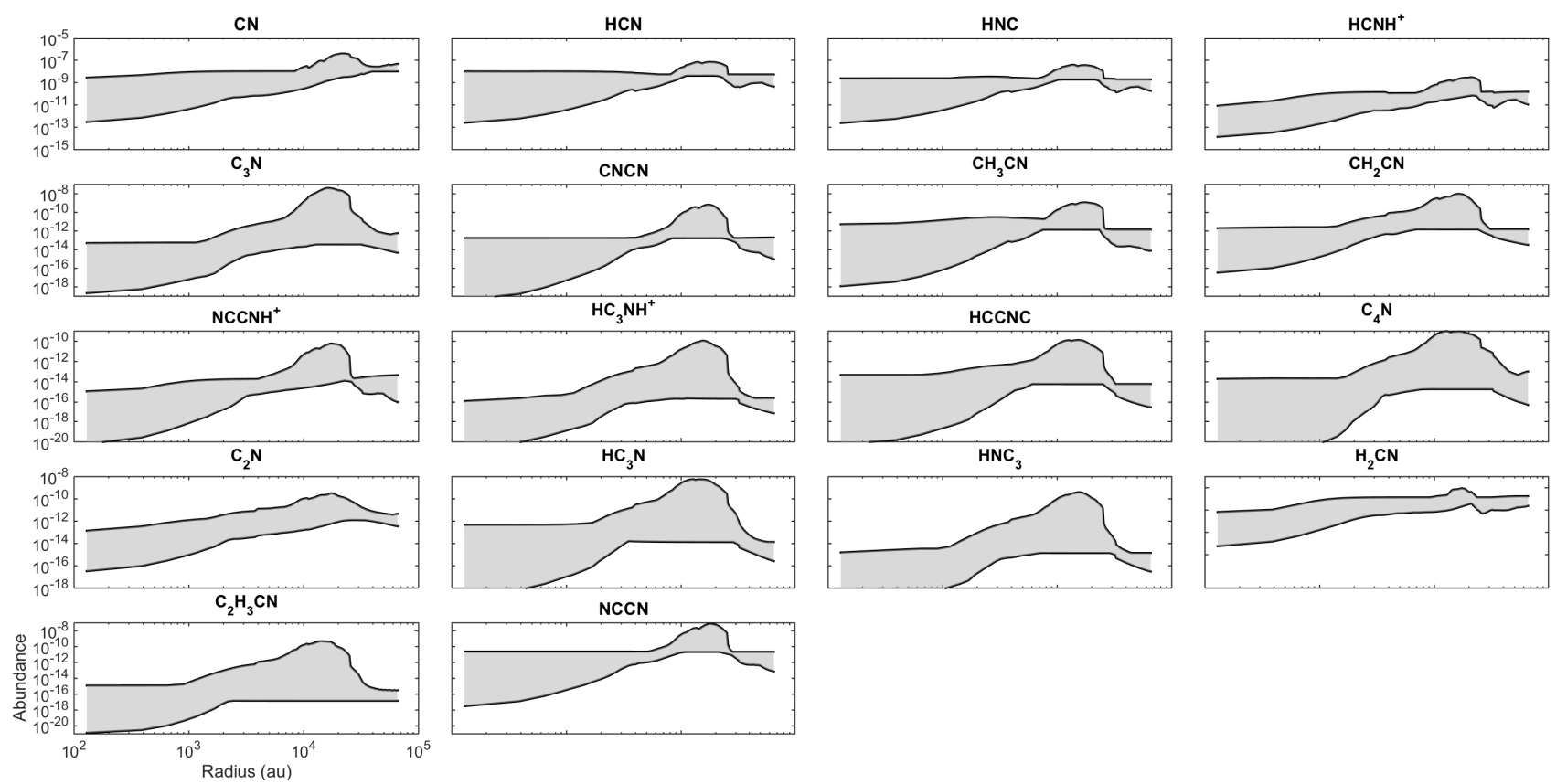

Fig. 7. Radial distribution of the nitrogen bearing species abundances in L1544 for an age between $10^{6}$ and $10^{7} \mathrm{yr}$.

Table 4. Initial gas phase elemental abundances assumed relative to the total nuclear hydrogen density $n_{\mathrm{H}}$.

\begin{tabular}{ll}
\hline \hline Species & \\
\hline $\mathrm{He}$ & $9.00 \times 10^{-2}$ \\
$\mathrm{~N}$ & $2.14 \times 10^{-5}$ \\
$\mathrm{O}$ & $1.76 \times 10^{-4}$ \\
$\mathrm{C}^{+}$ & $7.30 \times 10^{-5}$ \\
$\mathrm{~S}^{+}$ & $8.00 \times 10^{-8}$ \\
$\mathrm{Si}^{+}$ & $8.00 \times 10^{-9}$ \\
$\mathrm{Fe}^{+}$ & $3.00 \times 10^{-9}$ \\
$\mathrm{Na}^{+}$ & $2.00 \times 10^{-9}$ \\
$\mathrm{Mg}^{+}$ & $7.00 \times 10^{-9}$ \\
$\mathrm{Cl}^{+}$ & $1.00 \times 10^{-9}$ \\
$\mathrm{P}^{+}$ & $2.00 \times 10^{-10}$ \\
$\mathrm{~F}^{+}$ & $6.68 \times 10^{-9}$ \\
\hline
\end{tabular}

the NCCCN production, leading to a low but non-negligible rate constant at $10 \mathrm{~K}$. There is no experimental or theoretical data on the $\mathrm{C}+\mathrm{CNCN}$ reaction but considering the very high reactivity of carbon atom with unsaturated hydrocarbons and $\mathrm{HCN}$ and HNC (Loison \& Hickson 2015), which have similar -CN and -NC bonds than $\mathrm{CNCN}$, there are very little doubt, if any, that this reaction does not show any barrier. Moreover we do not find any barrier at DFT level (M06-2X/AVTZ) for the $\mathrm{C}$ attack on $\mathrm{CNCN}$ leading in a first step to CCNCN, CNCCN, and CNCNC adducts. These adducts can evolve in different ways. First there is always the back dissociation into $\mathrm{C}+\mathrm{CNCN}$, these back dissociation being important only if other bimolecular exit channels involve high transition states, which is the case of CCNCN. In that case radiative association may also play a minor role neglected here. For CNCCN and CNCNC adducts, the back dissociation will not be the main process as there are pathways leading to $\mathrm{C}+\mathrm{NCCN}$ channel. As not all the $\mathrm{C}+\mathrm{CNCN}$ entrance channels lead efficiently (without high TS on the pathway) to bimolecular products, we consider a smaller rate constant that the one given by capture theory for the $\mathrm{C}+\mathrm{CNCN} \rightarrow \mathrm{C}+$ $\mathrm{NCCN}$ reaction.

\subsection{Chemistry in the L1544 prestellar core}

We have applied the above chemistry to the prototypical prestellar source L1544. We used the gas-grain chemical code NAUTILUS (described in Ruaud et al. 2016) in its three-phase mode (gas-phase, grain-surface, and mantle chemistry) to predict the abundances of the simplest form of dicyanopolyynes species, $\mathrm{CNCN}$, and its related species, $\mathrm{CN}, \mathrm{NCCNH}^{+}, \mathrm{C}_{3} \mathrm{~N}$, $\mathrm{HCN}, \mathrm{HNC}, \mathrm{HCNH}^{+}, \mathrm{HC}_{3} \mathrm{NH}^{+}, \mathrm{HC}_{3} \mathrm{~N}, \mathrm{HNC}_{3}, \mathrm{HCCNC}, \mathrm{H}_{2} \mathrm{CN}$, $\mathrm{CH}_{3} \mathrm{CN}, \mathrm{CH}_{2} \mathrm{CN}, \mathrm{C}_{2} \mathrm{H}_{3} \mathrm{CN}$.

The methodology we have adopted here is similar to the studies already performed on L1544 (see for example Quénard et al. 2017a; Vastel et al. 2018a,b). A two step model has been used. The first phase represents the evolution of the chemistry in a diffuse or molecular cloud, with $T=20 \mathrm{~K}$ and several densities, ranging from $10^{2}$ to $2 \times 10^{4} \mathrm{~cm}^{-3}$. We present in Table 4 the values of the initial gas phase elemental abundances, based on the results found by Quénard et al. (2017a) and Vastel et al. (2018b). We then used the resulting abundances at $10^{6} \mathrm{yr}$ in a second phase model, taking into account the density and temperature structure of the L1544 prestellar core that shows evidence for gravitational contraction (Caselli et al. 2012; Keto et al. 2014). In order to compare the results from the chemical modelling (abundances with respect to $\mathrm{H}$ ) with the observations (column density) of the observed species presented in Sect. 2, we took into account the density profile across the core (see JiménezSerra et al. 2016; Quénard et al. 2017b; Vastel et al. 2018a,b) to determine the column density from the chemical modelling instead of the abundance at a specific radius. This methodology has been used for sources such as prestellar cores where molecular depletion at the centre, due to the low kinetic temperature and high $\mathrm{H}_{2}$ density, affects chemistry. Note that for cold dark clouds such as TMC-1, a single temperature and density have been assumed throughout the cloud, with a $\mathrm{H}_{2}$ column density of $\sim 10^{22} \mathrm{~cm}^{-2}$ (Cernicharo \& Guelin 1987; Fehér et al. 2016). 
CN
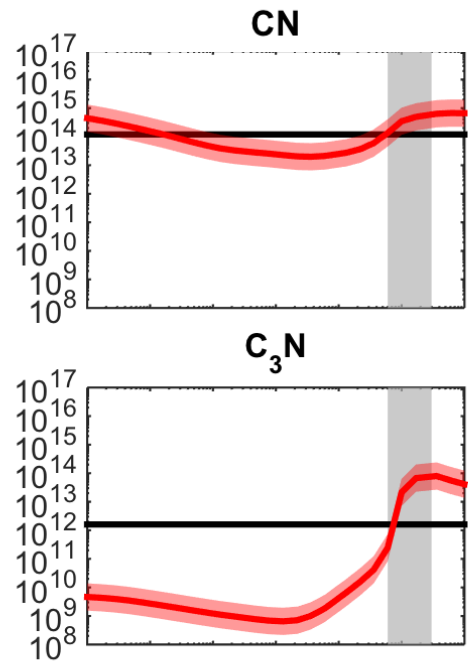

$\mathrm{NCCNH}^{+}$
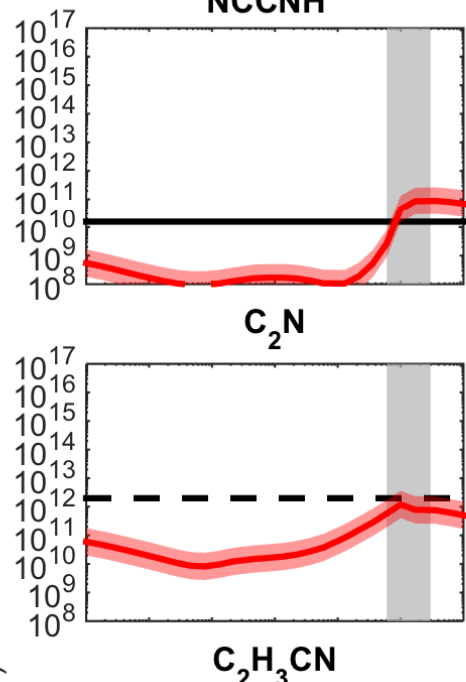

พิ
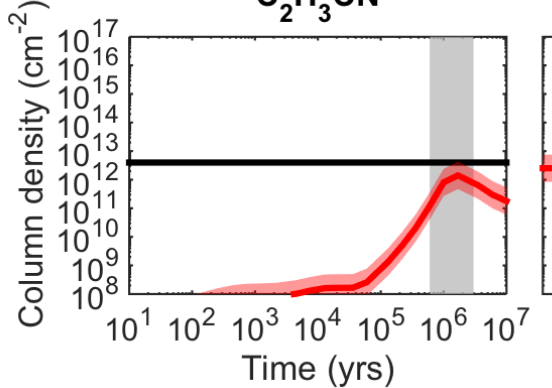

HCN

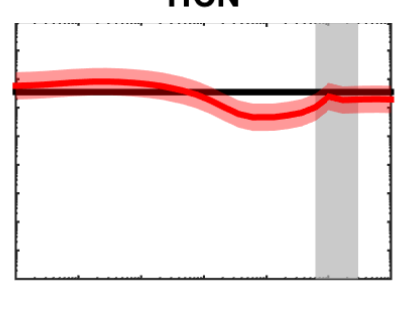

CNCN

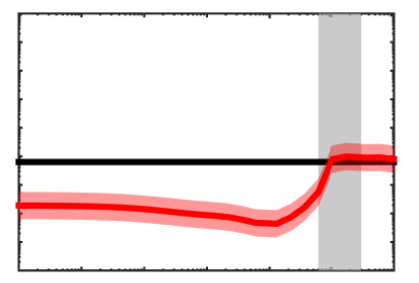

$\mathrm{HC}_{3} \mathrm{NH}^{+}$

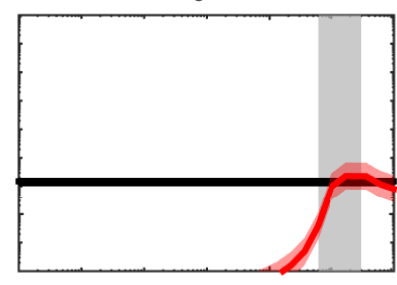

$\mathrm{HC}_{3} \mathrm{~N}$

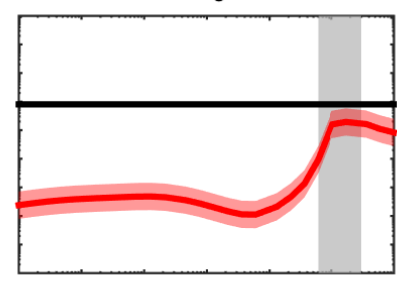

NCCN
HNC

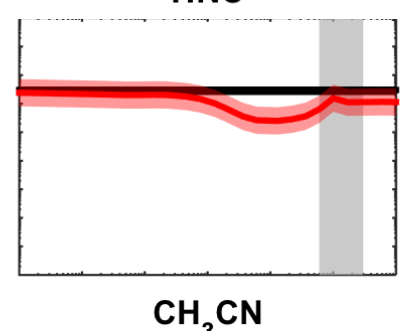

$\mathrm{CH}_{3} \mathrm{CN}$

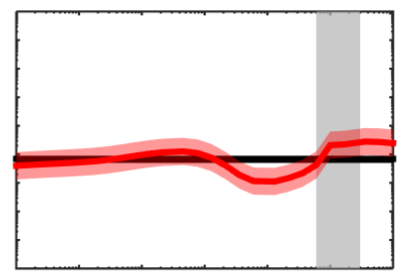

HCCNC

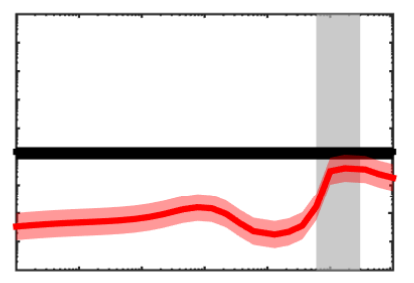

$\mathrm{HNC}_{3}$

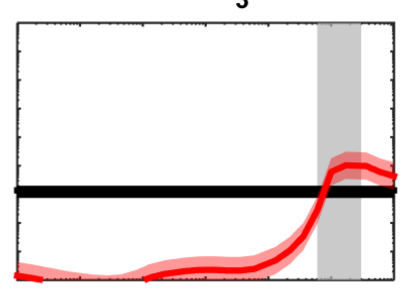

$\mathrm{HCNH}^{+}$
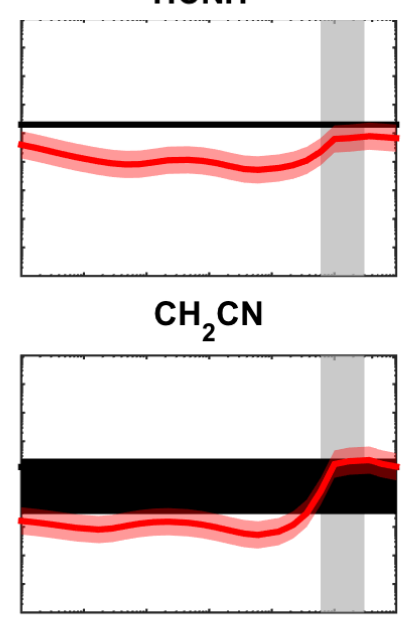

$\mathrm{C}_{4} \mathrm{~N}$

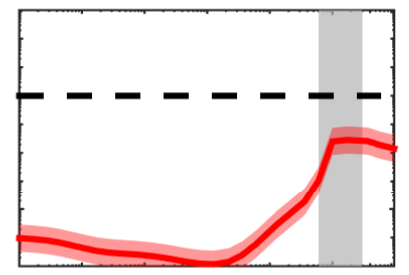

$\mathrm{H}_{2} \mathrm{CN}$

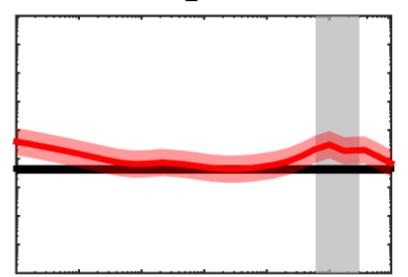

Fig. 8. Variation of the modelled column density (in red) as a function of time and the comparison with the observed column density (black horizontal lines). The dashed line corresponds to an upper limit for the column density. The thickness of the black line corresponds to the errors on the column density given in Table 2 . A variation by a factor of three on the modelled column density is shown in red. The grey vertical boxes highlight an area between $6 \times 10^{5}$ and $3 \times 10^{6} \mathrm{yr}$ (see text).

With the modifications of the chemical network presented in Sect. 4.1 we re-examine the chemistry of nitrogen species such as HCN, HNC (Quénard et al. 2017a), $\mathrm{HC}_{3} \mathrm{~N}$ (Quénard et al. 2017a; Hily-Blant et al. 2018), $\mathrm{HNC}_{3}$ (Vastel et al. 2018a), HCCNC (Vastel et al. 2018a), $\mathrm{HCNH}^{+}$(Quénard et al. 2017a), $\mathrm{HC}_{3} \mathrm{NH}+$ (Quénard et al. 2017a), $\mathrm{CH}_{2} \mathrm{CN}$ Vastel et al. (2015b).

We present in Fig. 7 the variation of the modelled abundance as a function of radius for ages between $10^{6}$ and $10^{7} \mathrm{yr}$, for an initial gas density of $3 \times 10^{3} \mathrm{~cm}^{-3}$. We checked that varying the initial density from $10^{2}$ to $2 \times 10^{4} \mathrm{~cm}^{-3}$ does not change much the results for an age between $10^{6}$ and $10^{7} \mathrm{yr}$. The abundances peak at a radius between [1-2] $\times 10^{4} \mathrm{au}$, which can be explained by the carbon, oxygen, and sulphur depletion in the cold and dense central regions of prestellar cores. However, this peak is less pronounced compared to other species that do not contain Nitrogen (see for example Vastel et al. 2018b, for the study of Sulphur bearing species). This can be explained by the fact that nitrogen remains present in the gas phase at densities well above and temperature well below that at which $\mathrm{CO}$ depletes on to grains. The resulting molecular differentiation has been seen in many starless cores (e.g. Kuiper et al. 1996; Tafalla et al. 1998, 2006; Ohashi et al. 1999; Spezzano et al. 2016). 
We present in Fig. 8 the variation of the modelled column density (in red) as a function of time from NAUTILUS and the comparison with the observed column densities (black horizontal lines) and upper limits (dashed horizontal lines) from Sect. 3. A variation by a factor of three on the modelled column density is shown. The thickness of the black lines corresponds to the uncertainty.

To compare the model results with observations, we used the method described in Wakelam et al. (2006) in which a distance of disagreement is computed as follows:

$D(\mathrm{t})=\frac{1}{n_{\mathrm{obs}}} \sum_{i}\left|\log (N(X))_{\mathrm{obs}, i}-\log (N(X))_{i}(\mathrm{t})\right|$,

where $N(X)_{\text {obs }, i}$ is the observed column density, $N(X)_{i}(\mathrm{t})$ is the modelled column density at a specific age and $n_{\mathrm{obs}}$ is the total number of observed species considered in this computation (14 in the case of nitrogen bearing species detected in L1544). We note that we did not take into account species where a lower limit has been derived from the observations $\left(\mathrm{C}_{2} \mathrm{~N}\right.$ and $\left.\mathrm{C}_{4} \mathrm{~N}\right)$.

Figure 9 shows its temporal variation, where a smaller value corresponds to a better agreement. Taking into account the present study, this distance is lower than unity for times higher than $6 \times 10^{5}$ and $3 \times 10^{6} \mathrm{yr}$. This time is of course model dependent and does not represent the "real" age of the source knowing that the chemical composition is constantly evolving in the interstellar medium. We then used the best-fit age $\left((1-3) \times 10^{6} \mathrm{yr}\right)$ from the similar work performed for Sulphur chemistry in L1544 (Vastel et al. 2018b) and pinpoint a range between $3 \times 10^{6}$ and $6 \times 10^{6} \mathrm{yr}$ for consistency between both studies.

We can see from Fig. 8 that all species are reproduced with the present chemical modelling. Using a higher elemental abundance for oxygen and/or carbon (higher by a factor of two, as observed in the diffuse medium, see for instance Jenkins 2009) will over-produce these species, leading to a much higher value for this distance of disagreement. NCCN reaches a calculated gas phase column density around $10^{14} \mathrm{~cm}^{-2}$ (and an abundance of $\sim 10^{-8}$ at $10^{4} \mathrm{au}$ ), similar to HCN. From our chemical modelling, the cyanogen/isocyanogen ratio is about a factor of 100 . The predicted abundance of NCCN on the grain surfaces remains however low $\left(\leq 10^{-12}\right)$ to be compared to the high grain surface $\mathrm{HCN}$ abundance of $\sim 5 \times 10^{-7}$. It can be also noted that in our calculations $\mathrm{C}_{2} \mathrm{~N}$ reaches a non negligible value, close to the upper limit which deserves to thorough investigation with more sensitive observations. The $\mathrm{C}_{2} \mathrm{H}_{3} \mathrm{CN}$ calculated abundance is slightly under estimated which is likely due to the fact that $\mathrm{C}_{2} \mathrm{H}_{4}$ is also under estimated in our modelling: $\mathrm{C}_{2} \mathrm{H}_{3} \mathrm{CN}$ is produced through the $\mathrm{CN}+\mathrm{C}_{2} \mathrm{H}_{4}$ reaction and is a good proxy of $\mathrm{C}_{2} \mathrm{H}_{4}$. Then, the gas phase and grain chemistry of $\mathrm{C}_{2} \mathrm{H}_{4}$ deserve to be reviewed as $\mathrm{C}_{2} \mathrm{H}_{4}$ is an important precursor of complex organic chemistry.

Our previous studies (Quénard et al. 2017a; Vastel et al. 2018a) tended to overestimate the production of $\mathrm{HCN}, \mathrm{HNC}$, $\mathrm{HC}_{3} \mathrm{~N}, \mathrm{HNC}_{3}, \mathrm{HC}_{3} \mathrm{NH}^{+}$. The inclusion of the new reactions, as well as the various KIDA updates revealed crucial to better understand the nitrogen chemistry, and nitriles in particular, in the cold and dense medium.

The study of the chemical evolution from the prestellar phase to the planetary disk is important to understand how the organics found in our own solar system have been produced. The comparison between the prestellar core phase and the content of the comets studied so far is an important Ariane thread to understand this link. Our study predict a large quantity of NCCN, unfortunately invisible in the rotational transitions tracing the cold medium. This linear symmetric molecule,

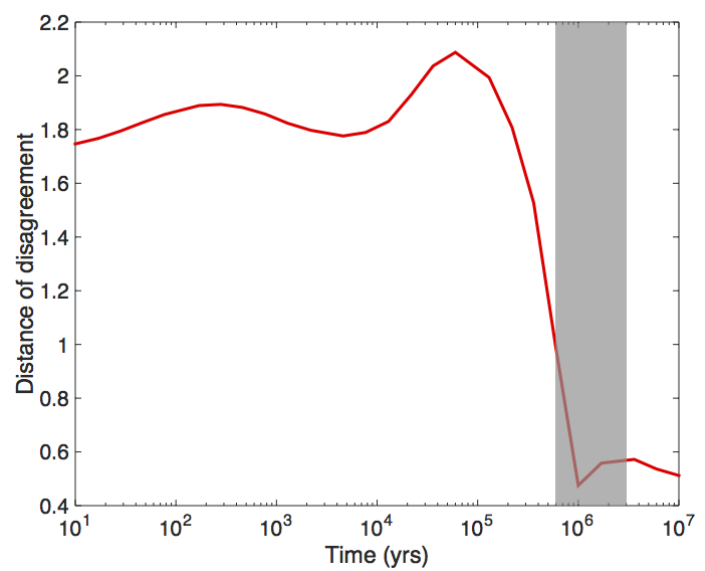

Fig. 9. Variation, in time, of the distance of disagreement. The grey box area represent a range between $6 \times 10^{5}$ and $3 \times 10^{6} \mathrm{yr}$ (see text).

without permanent dipole moment, has been suggested a long time ago (Swings \& Haser 1956) as a likely parent for the production of the $\mathrm{CN}$ radical observed in cometary atmospheres. Moreover, NCCN is likely the precursor of cyanomethanimine ( $\mathrm{HNCHCN}$ ), an HCN dimer proposed as precursor of adenine, recently detected by Rivilla et al. (2019) towards the Galactic Center quiescent molecular cloud $\mathrm{G}+0.693$. It is likely to have been formed on the surface of icy dust grains through the following reactions (Shivani \& Tandon 2017):

$\mathrm{s}-\mathrm{C}_{2} \mathrm{~N}_{2}+\mathrm{s}-\mathrm{H} \rightarrow \mathrm{s}-\mathrm{NCHCN}$,

$\mathrm{s}-\mathrm{NCHCN}+\mathrm{s}-\mathrm{H} \rightarrow \mathrm{s}-\mathrm{HNCHCN}$.

It therefore may be at the origin of a more complex cyanogen chemistry.

\section{Conclusions}

We investigated the formation of isocyanogen in the cold interstellar medium with the study of some nitrogen species in the prototypical prestellar core L1544. We report the detection of isocyanogen $(\mathrm{CNCN})$, protonated cyanogen $\left(\mathrm{NCCNH}^{+}\right)$, cyanoethynyl $\left(\mathrm{C}_{3} \mathrm{~N}\right)$, methylene amidogen $\left(\mathrm{H}_{2} \mathrm{CN}\right)$, methyl cyanide $\left(\mathrm{CH}_{3} \mathrm{CN}\right)$ and acrylonitrile $\left(\mathrm{C}_{2} \mathrm{H}_{3} \mathrm{CN}\right)$. We then built a detailed chemical network for the $\mathrm{NCCN} / \mathrm{CNCN} / \mathrm{HC}_{2} \mathrm{~N}_{2}^{+}$chemistry to understand the isocyanogen formation in the cold interstellar medium. The main cyanogen production pathways considered in this new network are the $\mathrm{CN}+\mathrm{HNC}$ and $\mathrm{N}+\mathrm{C}_{3} \mathrm{~N}$ reactions. The comparison between the observations of 14 detected nitrogen species in the $\mathrm{L} 1544$ prestellar core $(\mathrm{CN}, \mathrm{HCN}, \mathrm{HNC}$, $\mathrm{C}_{3} \mathrm{~N}, \mathrm{CNCN}, \mathrm{CH}_{3} \mathrm{CN}, \mathrm{CH}_{2} \mathrm{CN}, \mathrm{HCCNC}, \mathrm{HC}_{3} \mathrm{~N}, \mathrm{HNC}_{3}, \mathrm{H}_{2} \mathrm{CN}$, $\left.\mathrm{C}_{2} \mathrm{H}_{3} \mathrm{CN}, \mathrm{HCNH}^{+}, \mathrm{HC}_{3} \mathrm{NH}^{+}\right)$as well as two upper limits $\left(\mathrm{C}_{4} \mathrm{~N}\right.$, $\mathrm{C}_{2} \mathrm{~N}$ ) and the predictions from the chemical modelling shows a very good agreement. Although NCCN cannot be detected through its rotational spectrum (due to a lack of a permanent electric dipole moment), we can trace this species through the detection of $\mathrm{CNCN}$, since they are both produced through the same reaction. With this new chemical network, we predict a gas-phase column density of $\sim 10^{14} \mathrm{~cm}^{-2}$ for the "invisible" NCCN, with an expected cyanogen abundance greater than the isocyanogen abundance by a factor of 100 . However, its predicted abundance on the grain surfaces remains low compared to the high grain surface $\mathrm{HCN}$ abundance. Unless NCCN is efficiently 
formed within protoplanetary disks, it is an unlikely major source of $\mathrm{CN}$ in cometary coma through its photolysis.

Acknowledgements. We thank the CNRS program Physique et Chimie du Milieu Interstellaire (PCMI) co-funded by the Centre National d'Etudes Spatiales (CNES).

\section{References}

Agúndez, M., Cernicharo, J., de Vicente, P., et al. 2015, A\&A, 579, L10 Agúndez, M., Marcelino, N., \& Cernicharo, J. 2018, ApJ, 861, L22

Amano, T., \& Scappini, F. 1991, J. Chem. Phys., 95, 2280

Anicich, V. G. 2003, JPL publication, 03-19 NASA

Bockelee-Morvan, D., \& Crovisier, J. 1985, A\&A, 151, 90

Bockelée-Morvan, D., Lis, D. C., Wink, J. E., et al. 2000, A\&A, 353, 1101

Bonev, B., \& Komitov, B. 2000, BAAS, 32, 1072

Caselli, P., Keto, E., Bergin, E. A., et al. 2012, ApJ, 759, L37

Cazzoli, G., \& Puzzarini, C. 2006, J. Mol. Spectr., 240, 153

Cernicharo, J., \& Guelin, M. 1987, A\&A, 176, 299

Crutcher, R. M., Churchwell, E., \& Ziurys, L. M. 1984, ApJ, 283, 668

Dutuit, O., Carrasco, N., Thissen, R., et al. 2013, ApJS, 204, 20

Ehrenfreund, P., \& Charnley, S. B. 2000, ARA\&A, 38, 427

Fehér, O., Tóth, L. V., Ward-Thompson, D., et al. 2016, A\&A, 590, A75

Florescu-Mitchell, A. I., \& Mitchell, J. B. A. 2006, Phys. Rep., 430, 277

Fray, N., Bénilan, Y., Cottin, H., Gazeau, M.-C., \& Crovisier, J. 2005, Planet. Space Sci., 53, 1243

Geiss, J., Altwegg, K., Balsiger, H., \& Graf, S. 1999, Space Sci. Rev., 90, 253

Geppert, W. D., Ehlerding, A., Hellberg, F., et al. 2004, ApJ, 613, 1302

Gerry, M. C. L., Stroh, F., \& Winnewisser, M. 1990, J. Mol. Spectr., 140, 147

Gottlieb, C. A., Gottlieb, E. W., Thaddeus, P., \& Kawamura, H. 1983, ApJ, 275, 916

Guélin, M., Neininger, N., \& Cernicharo, J. 1998, A\&A, 335, L1

Hebrard, E., Dobrijevic, M., Loison, J. C., et al. 2013, A\&A, 552, A132

Herbst, E., Terzieva, R., \& Talbi, D. 2000, MNRAS, 311, 869

Hickson, K. M., Loison, J.-C., \& Wakelam, V. 2016, Chem. Phys. Lett., 659, 70

Hily-Blant, P., Walmsley, M., Pineau Des Forêts, G., \& Flower, D. 2008, A\&A, 480,5

Hily-Blant, P., Faure, A., Vastel, C., et al. 2018, MNRAS, 480, 1174

Hirota, T., Yamamoto, S., Mikami, H., \& Ohishi, M. 1998, ApJ, 503, 717

Irvine, W. M., Friberg, P., Hjalmarson, A., et al. 1988, ApJ, 334, 107

Jenkins, E. B. 2009, ApJ, 700, 1299

Jiménez-Serra, I., Vasyunin, A. I., Caselli, P., et al. 2016, ApJ, 830, L6

Kawaguchi, K., Ohishi, M., Ishikawa, S.-I., \& Kaifu, N. 1992a, ApJ, 386, L51

Kawaguchi, K., Takano, S., Ohishi, M., et al. 1992b, ApJ, 396, L49

Kawaguchi, K., Kasai, Y., Ishikawa, S.-I., et al. 1994, ApJ, 420, L95

Keto, E., Rawlings, J., \& Caselli, P. 2014, MNRAS, 440, 2616

Kolos, R., \& Grabowski, Z. 2000, Ap\&SS, 271, 65

Kuiper, T. B. H., Langer, W. D., \& Velusamy, T. 1996, ApJ, 468, 761

Lefloch, B., Bachiller, R., Ceccarelli, C., et al. 2018, MNRAS, accepted

Loison, J. C., \& Hickson, K. M. 2015, Chem. Phys. Lett., 635, 174

Loison, J.-C., Wakelam, V., Hickson, K. M., Bergeat, A., \& Mereau, R. 2014 MNRAS, 437, 930

Loison, J.-C., Agúndez, M., Marcelino, N., et al. 2016, MNRAS, 456, 4101

Loison, J.-C., Agúndez, M., Wakelam, V., et al. 2017, MNRAS, 470, 4075
McGonagle, D., \& Irvine, W. 1996, A\&A, 310, 970

Michael, J. V. 1980, Chem. Phys. Lett., 96, 129

Minh, Y., Irvine, W., Ohishi, M., et al. 1993, A\&A, 267, 229

Müller, H. S. P., Schlöder, F., Stutzki, J., \& Winnewisser, G. 2005, J. Mol. Spectr., 742, 215

Müller, H. S. P., Belloche, A., Menten, K. M., Comito, C., \& Schilke, P. 2008, J. Mol. Spectr., 251, 319

Ohashi, N., Lee, S., Wilner, D., \& Hayashi, M. 1999, ApJ, 518, 41

Ohishi, M., McGonagle, D., Irvine, William M., Yamamoto, S., \& Saito, S. 1994, ApJ, 427, L51

Osamura, Y., Fukuzawa, K., Terzieva, R., \& Herbst, E. 1999, ApJ, 519, 697

Petrie, S., \& Osamura, Y. 2004, J. Phys. Chem. A, 108, 3623

Petrie, S., Millar, T. J., \& Markwick, A. J. 2003, MNRAS, 341, 609

Plessis, S., Carrasco, N., Dobrijevic, M., \& Pernot, P. 2012, Icarus, 219, 254

Quénard, D., Vastel, C., Ceccarelli, C., et al. 2017a, MNRAS, 470, 3194

Quénard, D., Bottinelli, S., \& Caux, E. 2017b, MNRAS, 468, 685

Raksit, A. B., \& Bohme, D. K. 1984, Can. J. Chem., 62, 2123

Reiter, D., \& Janev, R. K. 2010, Contrib. Plasm. Phys., 50, 986

Rivilla, V. M., Martín-Pintado, J., Jiménez-Serra, I., et al. 2019, MNRAS, 483, 114

Ruaud, M., Wakelam, V., \& Hersant, F. 2016, MNRAS, 459, 3756

Safrany, D. R., \& Jaster, W. 1968a, J. Chem. Phys., 72, 3318

Safrany, D., \& Jaster, W. 1968b, J. Chem. Phys., 72, 3305

Safrany, D., \& Jaster, W. 1968c, J. Phys. Chem. A, 72, 3305

Schilke, P., Walmsley, C. M., Henkel, C., \& Millar, T. J. 1991, A\&A, 247, 487

Shivani, M., \& Tandon, P. 2017, Res. Astron. Astrophys., 17, 1

Spezzano, S., Bizzocchi, L., Caselli, P., Harju, J., \& Brünken, S. 2016, A\&A, 592, A11

Swings, P., \& Haser, L. 1956, Institut d'Astrophysique, Liège

Tafalla, M., Mardones, D., Myers, P. C., et al. 1998, ApJ, 504, 900

Tafalla, M., Santiago-García, J., Myers, P. C., et al. 2006, ApJ, 455, 577

Takano, S., Masuda, A., Hirahara, Y., et al. 1998, A\&A, 329, 1156

Teanby, N. A., Irwin, P. G. J., de Kok, R., et al. 2006, Icarus, 181, 243

Turner, B. E., Terzieva, R., \& Herbst, E. 1999, ApJ, 518, 699

Vastel, C., Ceccarelli, C., Lefloch, B., \& Bachiller, R. 2014, ApJ, 795, L2

Vastel, C., Bottinelli, S., Caux, E., Glorian, J.-M., \& Boiziot, M. 2015a, in SF2A2015: Proceedings of the Annual meeting of the French Society of Astronomy and Astrophysics, eds. F. Martins, S. Boissier, V. Buat, L. Cambrésy, \& P. Petit, 313

Vastel, C., Yamamoto, S., Lefloch, B., \& Bachiller, R. 2015b, A\&A, 582, L3

Vastel, C., Kawaguchi, K., Quénard, D., et al. 2018a, MNRAS, 474, L76

Vastel, C., Quénard, D., Le Gal, R., et al. 2018b, MNRAS, 478, 5514

Vidal, T. H. G., Loison, J.-C., Jaziri, A. Y., et al. 2017, MNRAS, 469, 435

Vigren, E., Semaniak, J., Hamberg, M., et al. 2012, Planet. Space Sci., 60, 102

Wakelam, V., Herbst, E., \& Selsis, F. 2006, A\&A, 451, 551

Wakelam, V., Smith, I. W. M., Herbst, E., et al. 2010, Space Sci. Rev., 156, 13

Wakelam, V., Herbst, E., Loison, J.-C., et al. 2012, ApJS, 199, 21

Wakelam, V., Loison, J.-C., Herbst, E., et al. 2015, ApJS, 217, 20

Wakelam, V., Loison, J.-C., Mereau, R., \& Ruaud, M. 2017, Mol. Astrophys., 6, 22

Whyte, A. R., \& Phillips, L. F. 1983, Chem. Phys. Lett., 98, 590

Yamamoto, S., \& Saito, S. 1992, J. Chem. Phys., 96, 4157

Zhao, Y., \& Truhlar, D. 2008, Theor. Chem. Acc., 120, 215 


\section{Appendix A: The $\mathrm{C}_{3} \mathrm{~N}_{2}$ system}

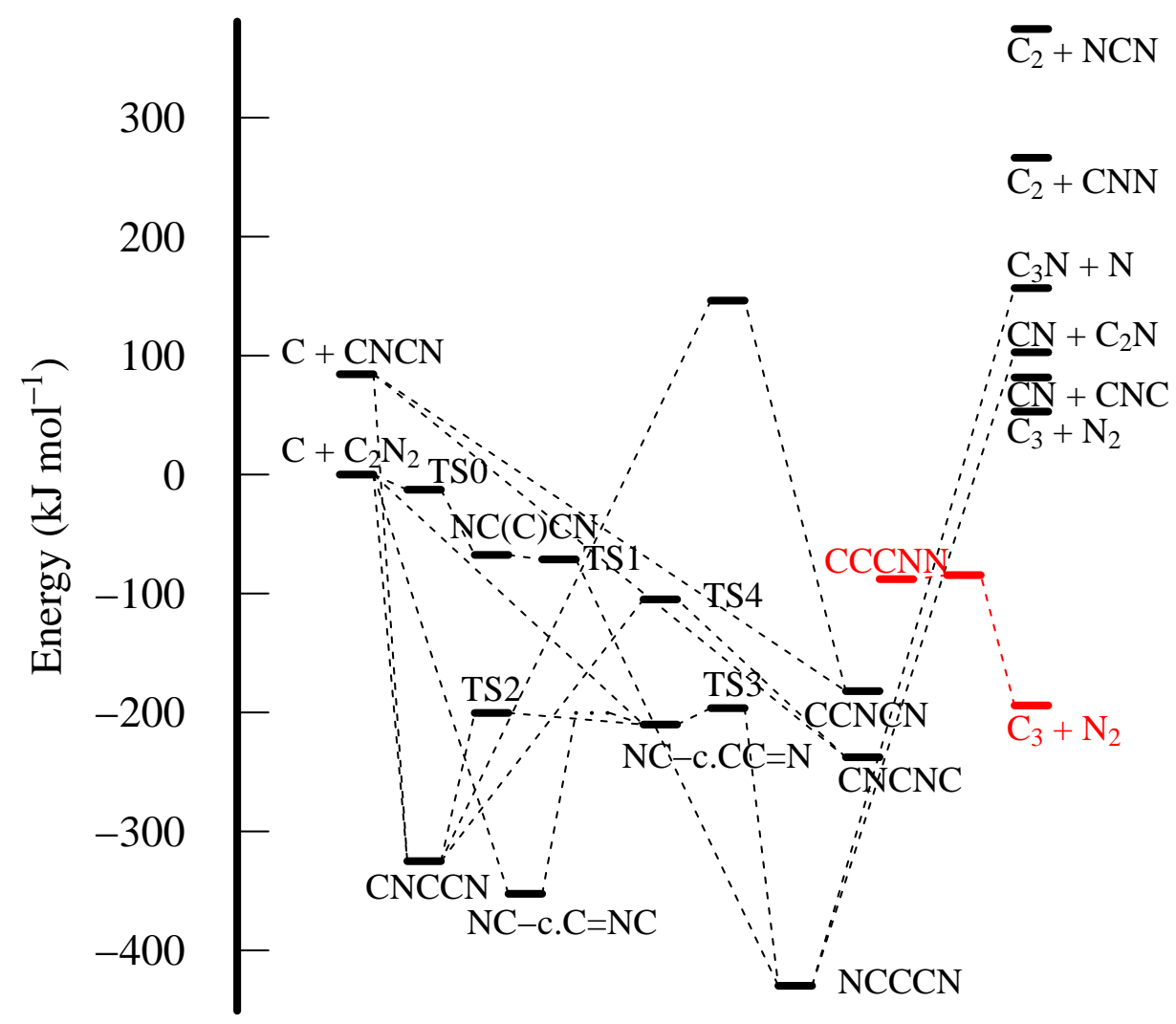

Fig. A.1. Partial potential energy diagram for the $\mathrm{C}_{3} \mathrm{~N}_{2}$ system on the triplet surface calculated at the M06-2X/AVTZ level including ZPE. The $\mathrm{C}_{3}+$ $\mathrm{N}_{2}$ exit channel in red is spin forbidden if $\mathrm{C}_{3}$ and $\mathrm{N}_{2}$ are in their singlet ground state. We cannot find the TS from NC-c.CC=N toward NC-c.C=NC at M06-2X/AVTZ level, both species having very similar structure (two different cyclic isomers with slightly different geometries and energies). $\mathrm{NC}-\mathrm{c} . \mathrm{CC}=\mathrm{N}$ is a local minimum but directly connected to NC-c.C=NC without barrier at semi-empirical AM1 level. So, this TS is very likely close in energy to $\mathrm{NC}-\mathrm{c} . \mathrm{CC}=\mathrm{N}$. 
Table A.1. Relative energies at the M06-2X/AVTZ level (in $\mathrm{kJ} \mathrm{mol}^{-1}$ at $0 \mathrm{~K}$ including ZPE) with respect to the $\mathrm{C}+\mathrm{NCCN}$ energy, geometries and frequencies (in $\mathrm{cm}^{-1}$, unscaled, calculated at the M06-2X/AVTZ level) of the various stationary points.

\begin{tabular}{|c|c|c|c|c|c|c|c|c|c|c|c|}
\hline $\begin{array}{c}\text { Species } \\
\text { (Energy, hartree) }\end{array}$ & $\begin{array}{c}\text { Relative } \\
\text { energies } \\
\left(\mathrm{kJ} \mathrm{mol}^{-1}\right)\end{array}$ & \multicolumn{3}{|c|}{ Geometries } & $\begin{array}{l}\text { Harmonic } \\
\text { Frequencies } \\
\left(\mathrm{cm}^{-1}\right)\end{array}$ & $\begin{array}{c}\text { Species } \\
\text { (Energy, hartree) }\end{array}$ & $\begin{array}{c}\text { Relative } \\
\text { energies } \\
\left(\mathrm{kJ} \mathrm{mol}^{-1}\right)\end{array}$ & \multicolumn{3}{|c|}{ Geometries } & $\begin{array}{l}\text { Harmonic } \\
\text { Frequencies } \\
\left(\mathrm{cm}^{-1}\right)\end{array}$ \\
\hline $\begin{array}{c}\mathrm{C} \\
(-37.8428462)\end{array}$ & \multirow[t]{2}{*}{0} & & & & & $\begin{array}{c}\mathrm{CNCNC} \\
(-223.571903) \\
0\end{array}$ & -237.6 & $\begin{array}{lc}\mathrm{C} & 0.000000 \\
\mathrm{~N} & 1.194279 \\
\mathrm{C} & 2.333831 \\
\mathrm{~N} & -1.194280 \\
\mathrm{C} & -2.333829\end{array}$ & $\begin{array}{r}0.550573 \\
0.024934 \\
-0.304378 \\
0.024941 \\
-0.304382\end{array}$ & $\begin{array}{l}0.0 \\
0.0 \\
0.0 \\
0.0 \\
0.0\end{array}$ & $\begin{array}{c}157,314 \\
344,362 \\
555,904 \\
1442,555 \\
\quad 904\end{array}$ \\
\hline $\begin{array}{c}\text { NCCN } \\
(-185.638541)\end{array}$ & & $\begin{array}{lll}\mathrm{C} & 0.0 & 0.0 \\
\mathrm{~N} & 0.0 & 0.0 \\
\mathrm{C} & 0.0 & 0.0 \\
\mathrm{~N} & 0.0 & 0.0\end{array}$ & $\begin{array}{r}0.693078 \\
1.839419 \\
-0.693078 \\
-1.839419\end{array}$ & & $\begin{array}{l}252,242 \\
556,556, \\
889,2331 \\
2520\end{array}$ & $\begin{array}{c}\begin{array}{c}\text { NC-c.CC=N } \\
(-223.564379)\end{array} \\
-\end{array}$ & -217.9 & $\begin{array}{ll}\text { C } & 0.277803 \\
\text { N } & 1.511956 \\
\text { C } & -1.049835 \\
\text { N } & -2.196347 \\
\text { C } & 1.570489\end{array}$ & $\begin{array}{c}-0.176986 \\
0.626681 \\
-0.036194 \\
0.063904 \\
-0.592501\end{array}$ & $\begin{array}{r}0.332143 \\
-0.046616 \\
0.027892 \\
-0.113744 \\
-0.172948\end{array}$ & $\begin{array}{c}178,209 \\
475,519 \\
581,695 \\
1258,1835 \\
2179\end{array}$ \\
\hline $\begin{array}{c}\text { TS0 } \\
(-223.486239)\end{array}$ & -12.8 & $\begin{array}{rr}\mathrm{C} & 0.000000 \\
\mathrm{~N} & 0.764346 \\
\mathrm{C} & -0.970230 \\
\mathrm{~N} & -1.780606 \\
\mathrm{C} & 2.155866\end{array}$ & $\begin{array}{r}0.580764 \\
1.443557 \\
-0.414400 \\
-1.220582 \\
-0.430857\end{array}$ & $\begin{array}{l}0.0 \\
0.0 \\
0.0 \\
0.0 \\
0.0\end{array}$ & $\begin{array}{c}95,248 \\
259,524 \\
557,883 \\
2292,2484 \\
165 \mathrm{i}^{*}\end{array}$ & $\begin{array}{c}\mathrm{NC}-\mathrm{c} . \mathrm{C}=\mathrm{NC} \\
(-223.615631)\end{array}$ & -352.5 & $\begin{array}{ll}\text { C } & 1.045657 \\
\text { N } & 2.193660 \\
\text { C }-1.635647 \\
\text { N }-1.383513 \\
\text { C }-0.355182\end{array}$ & $\begin{array}{r}-0.025774 \\
-0.158100 \\
-0.674838 \\
0.686246 \\
-0.098163\end{array}$ & $\begin{array}{l}0.0 \\
0.0 \\
0.0 \\
0.0 \\
0.0\end{array}$ & $\begin{array}{c}215,233 \\
547,594 \\
709,1091 \\
1351,1769 \\
2391\end{array}$ \\
\hline $\begin{array}{c}\text { TS1 } \\
(-223.508559)\end{array}$ & -71.3 & $\begin{array}{ll}\text { C } & 0.772759 \\
\text { N } & 1.902612 \\
\text { C } & -0.458613 \\
\text { N } & -1.586826 \\
\text { C } & -0.682563\end{array}$ & $\begin{array}{r}-0.127821 \\
-0.344216 \\
1.439100 \\
-0.856817 \\
0.098935\end{array}$ & $\begin{array}{l}0.0 \\
0.0 \\
0.0 \\
0.0 \\
0.0\end{array}$ & $\begin{array}{c}185,320 \\
437,524 \\
827,1088 \\
1321,2329 \\
269 \mathrm{i}\end{array}$ & $\begin{array}{c}\mathrm{CCNCN} \\
(-223.568679) \\
\theta=\Theta=-\Theta \theta\end{array}$ & -229.1 & $\begin{array}{ll}\text { C } & 0.720157 \\
\text { N } & 0.000000 \\
\text { C } & -0.689033 \\
\text { N } & -1.318285 \\
\text { C } & 1.506875\end{array}$ & $\begin{array}{c}1.052874 \\
0.090420 \\
-0.992394 \\
-1.964424 \\
2.125858\end{array}$ & $\begin{array}{l}0.0 \\
0.0 \\
0.0 \\
0.0 \\
0.0\end{array}$ & $\begin{array}{c}74,327,367, \\
470,473, \\
801,1418, \\
2099,2284\end{array}$ \\
\hline $\begin{array}{c}\text { TS2 } \\
(-223.560615)\end{array}$ & -208.0 & $\begin{array}{l}\mathrm{C}-0.212525 \\
\mathrm{~N}-1.433695 \\
\text { C } 1.077186 \\
\mathrm{~N} \quad 2.210179 \\
\mathrm{C}-1.770560\end{array}$ & $\begin{array}{c}-0.274800 \\
-0.468283 \\
-0.032867 \\
0.151706 \\
0.676216\end{array}$ & $\begin{array}{c}0.472400 \\
-0.244826 \\
0.047064 \\
-0.142576 \\
0.418100\end{array}$ & $\begin{array}{c}136,212 \\
454,542 \\
807,1330 \\
1809,2102 \\
249 \mathrm{i}\end{array}$ & & & & & & \\
\hline $\begin{array}{c}\text { TS3 } \\
(-223.556180)\end{array}$ & -196.4 & $\begin{array}{rr}\mathrm{C} & -1.678400 \\
\mathrm{~N} & -1.589409 \\
\mathrm{C} & 1.070842 \\
\mathrm{~N} & 2.202518 \\
\mathrm{C} & -0.185352\end{array}$ & $\begin{array}{r}-0.475090 \\
0.713136 \\
-0.078404 \\
0.213291 \\
-0.527337\end{array}$ & $\begin{array}{r}-0.183400 \\
0.024980 \\
0.011664 \\
-0.041803 \\
0.108796\end{array}$ & $\begin{array}{c}86,167 \\
429,463 \\
785,1420 \\
1889,2026 \\
574 \mathrm{i}\end{array}$ & & & & & & \\
\hline $\begin{array}{l}\text { TS4 } \\
(-223.521362) \\
==0\end{array}$ & -105.0 & $\begin{array}{rr}\mathrm{C} & -0.026645 \\
\mathrm{~N} & 0.092509 \\
\mathrm{C} & 0.930219 \\
\mathrm{~N} & -1.010132 \\
\mathrm{C} & -1.898784\end{array}$ & $\begin{array}{r}0.028609 \\
0.070552 \\
-0.094870 \\
-0.661401 \\
-1.225237\end{array}$ & $\begin{array}{r}-0.052280 \\
1.880259 \\
1.056185 \\
-0.527422 \\
-1.085129\end{array}$ & $\begin{array}{c}141,202, \\
371,468, \\
849,1379 \\
1919,1966, \\
640 \mathrm{i}\end{array}$ & & & & & & \\
\hline $\begin{array}{c}\text { NC(C)CN } \\
(-223.507120) \\
-\end{array}$ & -68.6 & $\begin{array}{l}\text { C } 0.882620 \\
\text { N } 2.028775 \\
\text { C }-0.557487 \\
\text { C }-1.132394 \\
\text { N }-1.336837\end{array}$ & $\begin{array}{r}-0.030559 \\
-0.055695 \\
0.032441 \\
1.277759 \\
-1.041141\end{array}$ & $\begin{array}{l}0.0 \\
0.0 \\
0.0 \\
0.0 \\
0.0\end{array}$ & $\begin{array}{c}144,226, \\
287,521, \\
569,811, \\
1050,1364, \\
2405\end{array}$ & & & & & & \\
\hline $\begin{array}{c}\text { NCCCN } \\
(-223.647236) \\
=-0=\end{array}$ & -435.4 & $\begin{array}{ll}\text { C } & 1.319939 \\
\text { N } & 2.493516 \\
\text { C } & -1.319939 \\
\text { N } & -2.493516 \\
\text { C } & 0.000000\end{array}$ & $\begin{array}{cc}0.1232 & 0 \\
-0.1309 & 0 \\
0.1105 & 0 \\
-0.1550 & 0 \\
0.0998 & 0\end{array}$ & $\begin{array}{l}0.0 \\
0.0 \\
0.0 \\
0.0 \\
0.0\end{array}$ & $\begin{array}{c}71,346, \\
425,431, \\
431,723, \\
1541,1814, \\
2047\end{array}$ & & & & & & \\
\hline $\begin{array}{c}\mathrm{CNCCN} \\
(-223.608042) \\
=-\end{array}$ & -332.5 & $\begin{array}{ll}\text { C } & -0.057602 \\
\text { N } & -1.278625 \\
\text { C } & 1.225118 \\
\text { N } & 2.366825 \\
\text { C } & -2.437082\end{array}$ & $\begin{array}{c}0.433435 \\
0.022854 \\
0.030247 \\
-0.209296 \\
-0.246167\end{array}$ & $\begin{array}{l}0.0 \\
0.0 \\
0.0 \\
0.0 \\
0.0\end{array}$ & $\begin{array}{c}132,350 \\
361,422, \\
508,785, \\
1504,1957, \\
2073\end{array}$ & & & & & & \\
\hline
\end{tabular}

Notes. The absolute energies at the M06-2X/AVTZ level including ZPE in hartree are also given in Col. 1. The i label represents an imaginary frequency which is characteristic of transition state (TS). The TS is the structure with the highest potential energy along the reaction coordinate. $\mathrm{NC}-\mathrm{c} . \mathrm{CC}=\mathrm{N}$ and $\mathrm{NC}-\mathrm{c} . \mathrm{C}=\mathrm{NC}$ are two different cyclic isomers with slightly different geometries and energies. 
Appendix B: The NCCCN $\rightarrow \mathrm{TS} 1 \rightarrow \mathrm{NC}(\mathrm{C}) \mathrm{CN}$ isomerization
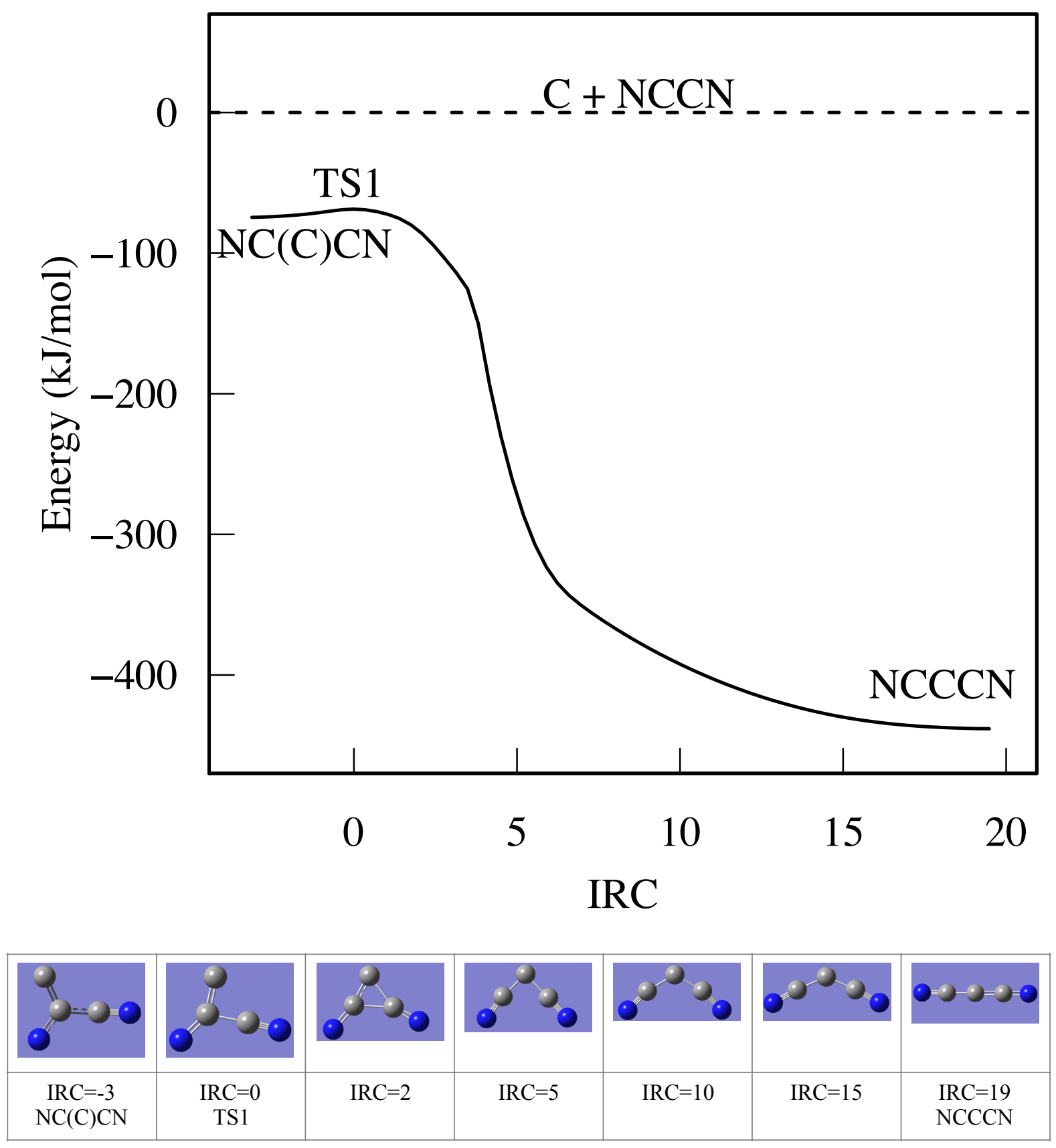

Fig. B.1. Intrinsic reaction coordinate (minimum energy path) at M06-2X/cc-pVTZ for the NCCCN $\rightarrow$ TS1 $\rightarrow$ NC(C)CN isomerization. 


\section{Appendix C: The CNCCN $\rightarrow$ NCCCN isomerization}

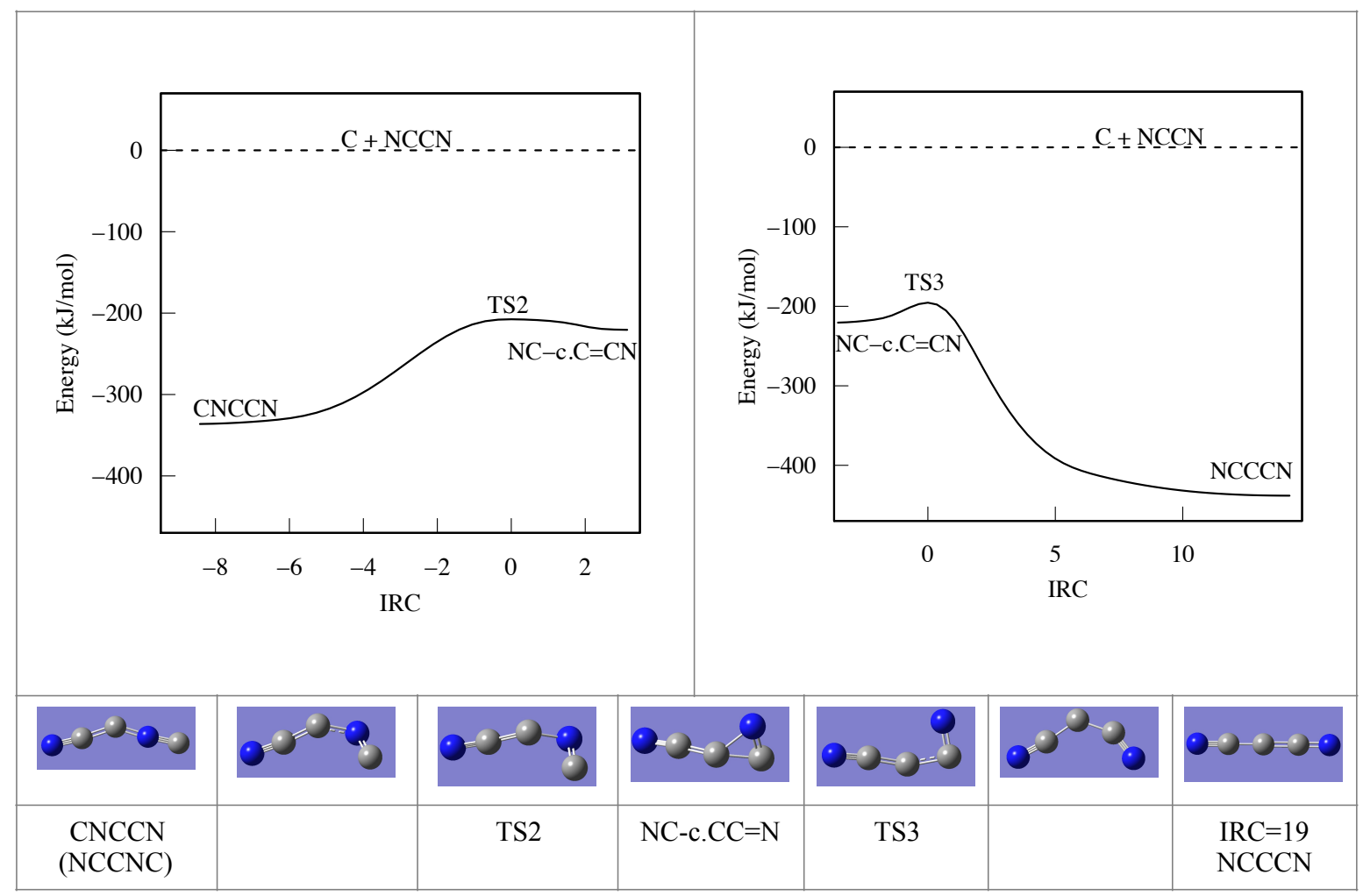

Fig. C.1. Intrinsic reaction coordinate (minimum energy path) at M06-2X/cc-pVTZ for the CNCCN $\rightarrow$ NCCCN isomerization.

\section{Appendix D: The NCCN $\rightarrow$ CNCN isomerization}

Table D.1. Relative energies at the M06-2X/AVTZ level (in $\mathrm{kJ} \mathrm{mol}^{-1}$ at $0 \mathrm{~K}$ including ZPE) with respect to the NCCN energy, geometries and frequencies (in $\mathrm{cm}^{-1}$, unscaled, calculated at the M06-2X/AVTZ level) of the various stationary points for the NCCN $\rightarrow \mathrm{CNCN}$ isomerization.

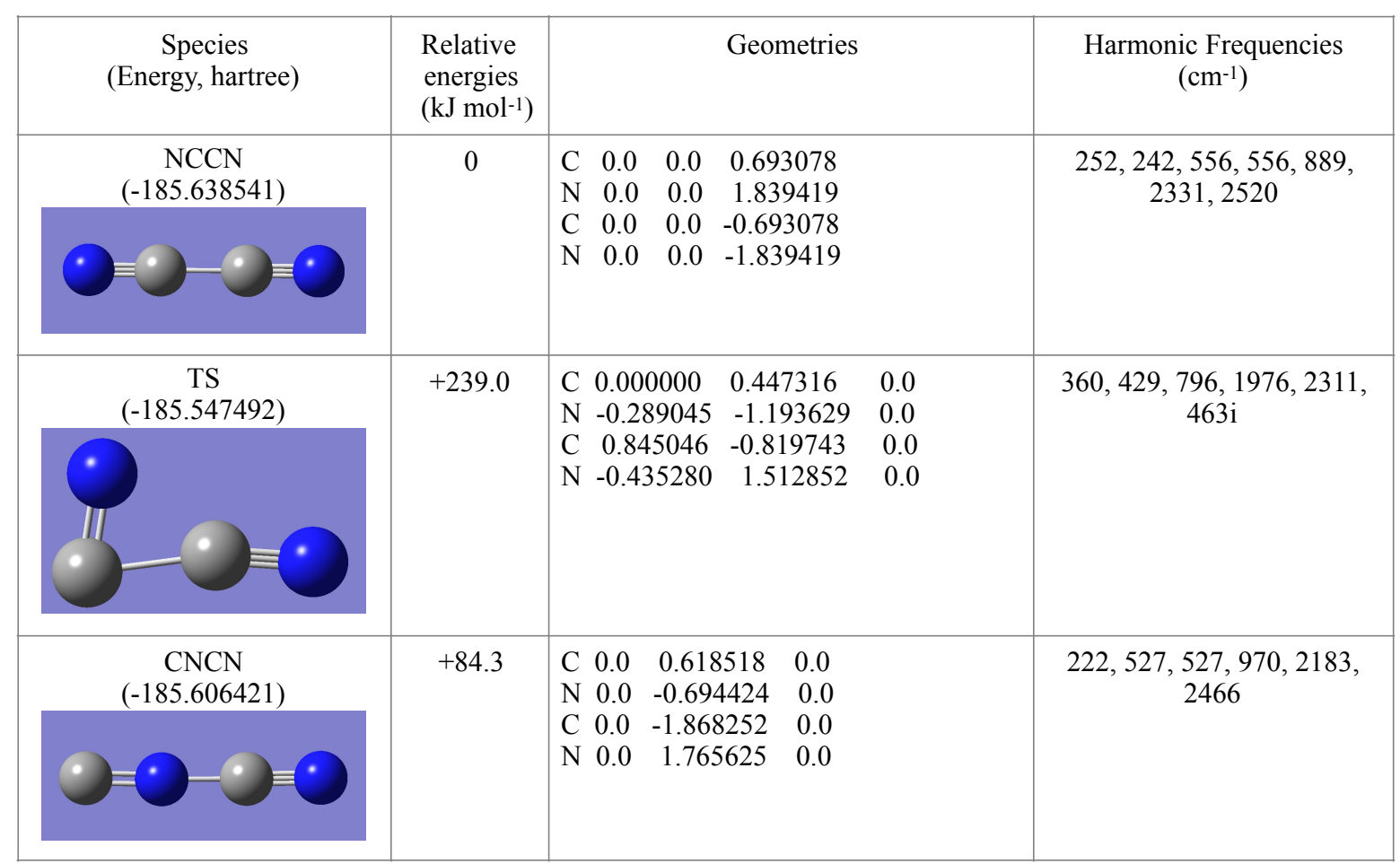

Notes. The absolute energies at the M06-2X/AVTZ level including ZPE in hartree are also given in Col. 1. The i label represents an imaginary frequency which is characteristic of transition state. TS is the structure with the highest potential energy along the reaction coordinate. 
A\&A 625, A91 (2019)

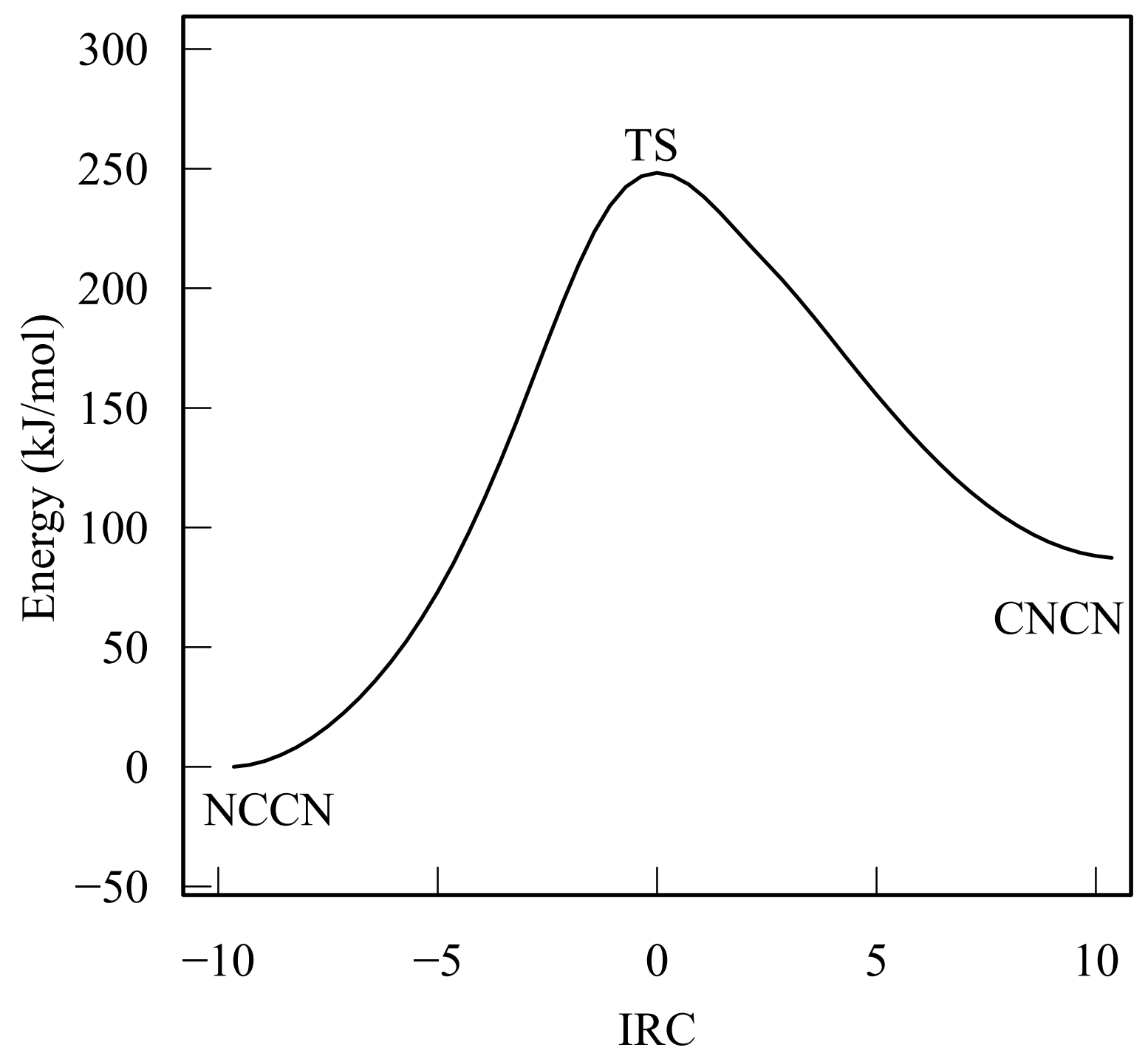

Fig. D.1. Intrinsic reaction coordinate pathway for the $\mathrm{NCCN} \rightarrow \mathrm{CNCN}$ isomerization calculated at M06-2X/AVTZ level. 


\section{Appendix E: N, O + NCCN}

Table E.1. Relative energies at the M06-2X/AVTZ level (in kJ mol ${ }^{-1}$ at $0 \mathrm{~K}$ including ZPE) with respect to the $\mathrm{N}+\mathrm{NCCN}$ and $\mathrm{O}+\mathrm{NCCN}$ energies, geometries and frequencies (in $\mathrm{cm}^{-1}$, unscaled, calculated at the M06-2X/AVTZ level) of the various transition states for $\mathrm{N}$ and $\mathrm{O}$ atoms additions on NCCN.

\begin{tabular}{|c|c|c|c|c|c|}
\hline $\begin{array}{c}\text { Species } \\
\text { (Energy, hartree) }\end{array}$ & $\begin{array}{l}\text { Relative } \\
\text { energies } \\
\left(\mathrm{kJ} \mathrm{mol}^{-1}\right)\end{array}$ & \multicolumn{3}{|c|}{ Geometries } & $\begin{array}{l}\text { Harmonic Frequencies } \\
\qquad\left(\mathrm{cm}^{-1}\right)\end{array}$ \\
\hline $\mathrm{TSa}(\mathrm{N}+\mathrm{NCCN})$ & +90.8 & $\begin{array}{lr}\mathrm{C} & -1.237314 \\
\mathrm{~N} & -2.333194 \\
\mathrm{C} & 0.038798 \\
\mathrm{~N} & 1.211928 \\
\mathrm{~N} & 2.148566\end{array}$ & $\begin{array}{r}-0.890800 \\
0.350486 \\
-0.505650 \\
-0.673190 \\
0.763754\end{array}$ & $\begin{array}{c}0.0 \\
0.0 \\
0.0 \\
0.0 \\
0.0\end{array}$ & $\begin{array}{c}86,266,389,5,587,885 \\
2030,2330 \\
746 \mathrm{i}\end{array}$ \\
\hline $\mathrm{TSb}(\mathrm{N}+\mathrm{NCCN})$ & +62.8 & $\begin{array}{lr}\mathrm{C} & -0.912386 \\
\mathrm{~N} & -2.048315 \\
\mathrm{C} & 0.491200 \\
\mathrm{~N} & 1.397924 \\
\mathrm{~N} & 1.012426\end{array}$ & $\begin{array}{r}-0.154363 \\
-0.537500 \\
-0.312928 \\
-1.066799 \\
1.472709\end{array}$ & $\begin{array}{l}0.0 \\
0.0 \\
0.0 \\
0.0 \\
0.0\end{array}$ & $\begin{array}{c}180,277,343,514,569 \\
865,2012,2434 \\
673 \mathrm{i}\end{array}$ \\
\hline $\mathrm{TSa}(\mathrm{O}+\mathrm{NCCN})$ & +77.8 & $\begin{array}{lr}\mathrm{C} & 1.319954 \\
\mathrm{~N} & 2.440898 \\
\mathrm{C} & 0.000000 \\
\mathrm{~N} & -1.164074 \\
\mathrm{O} & -2.107186\end{array}$ & $\begin{array}{c}0.062175 \\
-0.204376 \\
0.419191 \\
0.605296 \\
-0.711830\end{array}$ & $\begin{array}{c}0.0 \\
0.0 \\
0.0 \\
0.0 \\
0.0\end{array}$ & $\begin{array}{c}102,260,392,497,564 \\
887,2045,2346 \\
752 \mathrm{i}\end{array}$ \\
\hline $\mathrm{TSb}(\mathrm{O}+\mathrm{NCCN})$ & +39.7 & $\begin{array}{lc}\mathrm{C} & -1.713700 \\
\mathrm{~N} & -2.129137 \\
\mathrm{C} & 0.361401 \\
\mathrm{~N} & 1.299567 \\
\mathrm{O} & 1.210175\end{array}$ & $\begin{array}{r}-0.108396 \\
0.120855 \\
-0.407788 \\
-1.108759 \\
1.251554\end{array}$ & $\begin{array}{l}0.0 \\
0.0 \\
0.0 \\
0.0 \\
0.0\end{array}$ & $\begin{array}{c}177,270,320,511,573,863 \\
2137,2456 \\
654 \mathrm{i}\end{array}$ \\
\hline
\end{tabular}

Notes. The i label represents an imaginary frequency which is characteristic of transition state. 


\section{Appendix F: $\mathrm{H}, \mathrm{N}, \mathrm{O}+\mathrm{CNCN}$}

Table F.1. Relative energies at the M06-2X/AVTZ level (in kJ mol ${ }^{-1}$ at $0 \mathrm{~K}$ including ZPE) with respect to the $\mathrm{H}+\mathrm{CNCN}, \mathrm{N}+\mathrm{CNCN}$ and $\mathrm{O}+$ $\mathrm{CNCN}$ energies, geometries and frequencies (in $\mathrm{cm}^{-1}$, unscaled, calculated at the M06-2X/AVTZ level) of the various transition states for $\mathrm{H}, \mathrm{N}$, and $\mathrm{O}$ atoms additions on $\mathrm{CNCN}$.

\begin{tabular}{|c|c|c|c|c|c|}
\hline $\begin{array}{c}\text { Species } \\
\text { (Energy, hartree) }\end{array}$ & $\begin{array}{l}\text { Relative } \\
\text { energies } \\
\left(\mathrm{kJ} \mathrm{mol}^{-1}\right)\end{array}$ & \multicolumn{3}{|c|}{ Geometries } & $\begin{array}{l}\text { Harmonic Frequencies } \\
\qquad\left(\mathrm{cm}^{-1}\right)\end{array}$ \\
\hline $\mathrm{TS}(\mathrm{H}+\mathrm{CNCN})$ & +12.7 & $\begin{array}{cc}\mathrm{C} & -0.169164 \\
\mathrm{~N} & 0.000000 \\
\mathrm{~N} & -0.319780 \\
\mathrm{C} & 0.172505 \\
\mathrm{H} & 2.218409\end{array}$ & $\begin{array}{c}-0.699869 \\
0.631800 \\
-1.837872 \\
1.763921 \\
2.278571\end{array}$ & $\begin{array}{c}0.0 \\
0.0 \\
0.0 \\
0.0 \\
0.0\end{array}$ & $\begin{array}{c}168,224,3,522,531,971 \\
2159,2456 \\
575 \mathrm{i}\end{array}$ \\
\hline $\mathrm{TS}(\mathrm{N}+\mathrm{CNCN})$ & +31.6 & $\begin{array}{cr}\mathrm{C} & 1.221262 \\
\mathrm{~N} & -0.011110 \\
\mathrm{C} & -1.146256 \\
\mathrm{~N} & 2.312717 \\
\mathrm{~N} & -2.365898\end{array}$ & $\begin{array}{r}-0.010675 \\
0.416224 \\
0.767425 \\
-0.373950 \\
-0.690917\end{array}$ & $\begin{array}{c}-0.23 \\
0.00 \\
0.92 \\
0.48 \\
-0.01\end{array}$ & $\begin{array}{c}67,228,326,494,540,975 \\
2082,2420 \\
452 \mathrm{i}\end{array}$ \\
\hline $\mathrm{TS}(\mathrm{O}+\mathrm{CNCN})$ & +7.8 & $\begin{array}{cc}\mathrm{C} & 1.308108 \\
\mathrm{~N} & 0.000000 \\
\mathrm{~N} & 2.453779 \\
\mathrm{C} & -1.171514 \\
\mathrm{O} & -2.249502\end{array}$ & $\begin{array}{r}0.331553 \\
0.416304 \\
0.260601 \\
0.510500 \\
-1.216035\end{array}$ & $\begin{array}{r}0.0 \\
0.0 \\
0.0 \\
0.0 \\
0.0\end{array}$ & $\begin{array}{c}86,215,263,519,524,971 \\
2165,2454 \\
344 \mathrm{i}\end{array}$ \\
\hline
\end{tabular}

Notes. The i label represents an imaginary frequency which is characteristic of transition state. 


\section{Appendix G: The $\mathrm{CN}+\mathrm{HCNH}^{+}$reaction}

Table G.1. Relative energies at the M06-2X/AVTZ level (in kJ/ mol ${ }^{-1}$ at $0 \mathrm{~K}$ including ZPE) with respect to the $\mathrm{CN}+\mathrm{HCNH}{ }^{+}$energy, geometries and frequencies (in $\mathrm{cm}^{-1}$, unscaled, calculated at the M06-2X/AVTZ level) of the various stationary points for the $\mathrm{CN}+\mathrm{HCNH}^{+}$reaction.

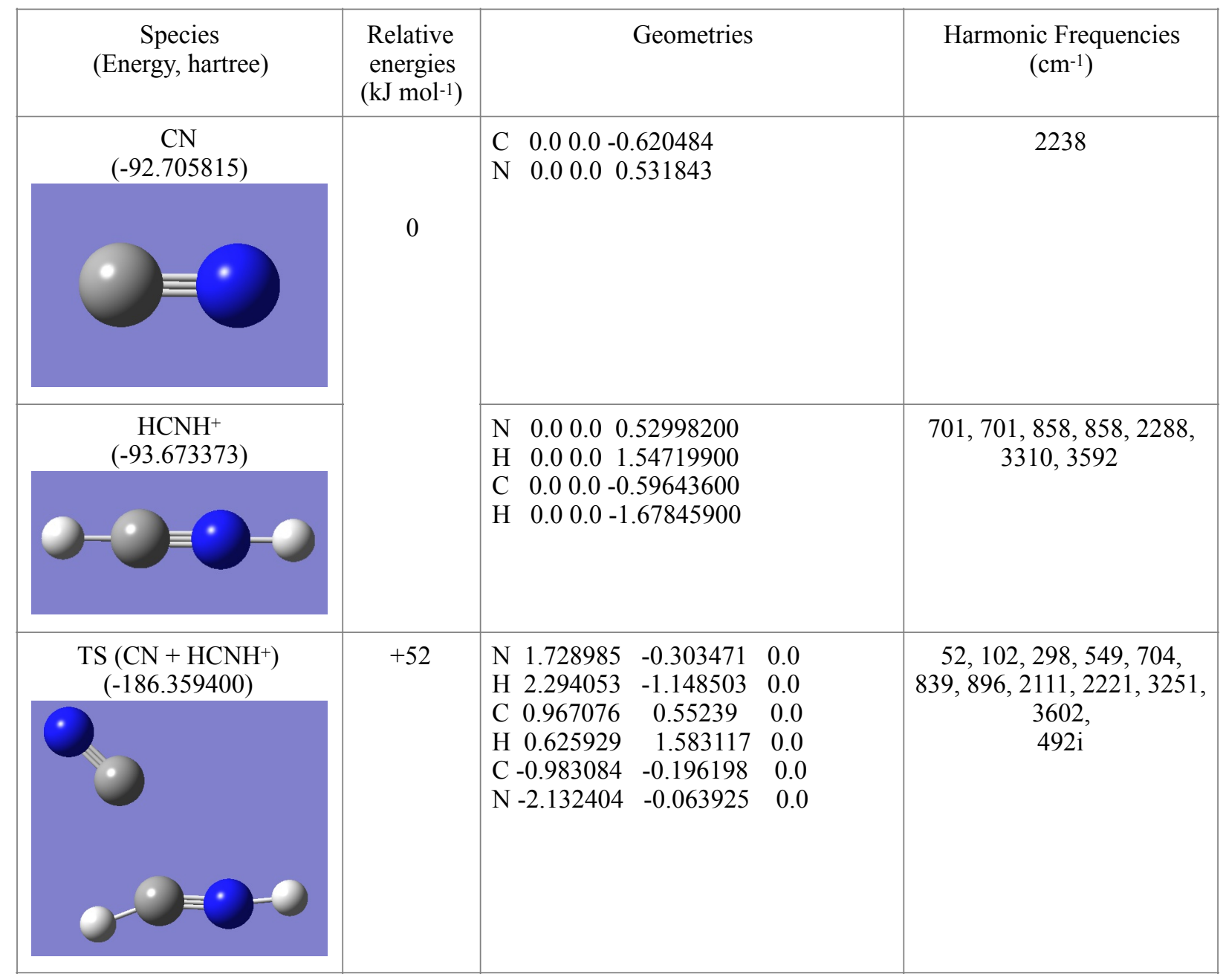

Notes. The absolute energies at the M06-2X/AVTZ level including ZPE in hartree are also given in Col. 1. The i label represents an imaginary frequency which is characteristic of transition state. 